\title{
Large scale structure in the SDSS galaxy survey
}

\author{
A. Doroshkevich ${ }^{1,2}$, D. L. Tucker ${ }^{3}$, S. Allam ${ }^{3,4}$, and M. J. Way ${ }^{1,5}$ \\ 1 Theoretical Astrophysics Center, Juliane Maries Vej 30, 2100 Copenhagen $\emptyset$, Denmark \\ 2 Keldysh Institute of Applied Mathematics, Russian Academy of Sciences, 125047 Moscow, Russia \\ 3 Fermi National Accelerator Laboratory, MS 127, PO Box 500, Batavia, IL 60510 USA \\ ${ }^{4}$ Department of Astronomy, New Mexico State University, Las Cruces, NM 88003-8001 USA \\ 5 NASA Ames Research Center, Space Sciences Division, MS 245-6, Moffett Field, CA 94035, USA
}

Received 15 July 2003 / Accepted 23 December 2003

\begin{abstract}
The Large Scale Structure in the galaxy distribution is investigated using Data Release 1 of the Sloan Digital Sky Survey. Using the Minimal Spanning Tree technique we have extracted sets of filaments, of wall-like structures, of galaxy groups, and of rich clusters from this unique sample. The physical properties of these structures were then measured and compared with the statistical expectations based on Zel'dovich theory.

The measured characteristics of galaxy walls were found to be consistent with those for a spatially flat $\Lambda$ CDM cosmological model with $\Omega_{m} \approx 0.3$ and $\Omega_{\Lambda} \approx 0.7$, and for Gaussian initial perturbations with a Harrison - Zel'dovich power spectrum. Furthermore, we found that the mass functions of groups and of unrelaxed structure elements generally fit well with the expectations from Zel'dovich theory. We also note that both groups and rich clusters tend to prefer the environments of walls, which tend to be of higher density, rather than the environments of filaments, which tend to be of lower density.
\end{abstract}

Key words. cosmology: large-scale structure of the Universe - surveys

\section{Introduction}

With the advent of the Durham/UKST Galaxy Redshift Survey (DURS, Ratcliffe et al. 1996) and the Las Campanas Redshift Survey (LCRS, Shectman et al. 1996), the galaxy distribution on scales up to $\sim 300 \mathrm{~h}^{-1} \mathrm{Mpc}$ could be studied. Now these investigations can be extended using the public data sets from the Sloan Digital Sky Survey's Data Release 1 (SDSS DR1, Stoughton et al. 2002; Abazajian et al. 2003), which contains redshifts for $\approx 100000$ galaxies in four slices for distances $D \leq$ $600 \mathrm{~h}^{-1} \mathrm{Mpc}$.

The analysis of the spatial galaxy distribution in the DURS and the LCRS has revealed that the Large Scale Structure (LSS) is composed of walls and filaments, that galaxies are divided roughly equally into each of these two populations (with few or no truly isolated galaxies), and that richer walls are linked to the joint random network of the cosmic web by systems of filaments (Doroshkevich et al. 2000, 2001). Furthermore, these findings are consistent with results obtained for simulations of dark matter (DM) distributions (see e.g. Cole et al. 1998; Jenkins et al. 1998) and for mock galaxy catalogues based upon DM simulations (Cole et al. 1998).

The quantitative statistical description of the LSS is in itself an important problem. Beyond that, though, the analysis of rich catalogues can also provide estimates for certain

Send offprint requests to: D. L. Tucker, e-mail: dtucker@fnal.gov cosmological parameters and for the characteristics of the initial power spectrum of perturbations. To do so, some theoretical models of structure formation can be used.

The close connection between the LSS and Zel'dovich pancakes has been discussed by Thompson \& Gregory (1978) and by Oort (1983). Now this connection is verified by the comparison of the statistical characteristics of observed and simulated walls with theoretical expectations (Demiański \& Doroshkevich 1999, 2002, hereafter DD99 and DD02) based on the Zel'dovich theory of nonlinear gravitational instability (Zel'dovich 1970; Shandarin \& Zeldovich 1989). This approach connects the characteristics of the LSS with the main parameters of the underlying cosmological scenario and the initial power spectrum, and it permits the estimation of some of these parameters using the measured properties of walls. It was examined with the simulated DM distribution (DD99; Demiański et al. 2000), and was found that, for sufficiently representative samples of walls, a precision of better than $20 \%$ can be reached.

Effective methods of the statistical description of the LSS based on the Minimal Spanning Tree (MST) technique were developed by Demiański et al. (2000) and Doroshkevich et al. (2000, 2001), who applied them to DM simulations and to the DURS and the LCRS. These methods introduced in Barrow et al. (1985) and van de Weygaert (1991) generalize the popular "friends-of-friends" approach. In this paper we apply the same approach to the SDSS DR1, a sample from which we can obtain 
more representative and more precise measures of the properties of the LSS and the initial power spectrum of perturbations. Alternative methods based on Minkowski functionals are proposed by, for example, Schmalzing et al. (1999), or Sheth et al. (2002)

With the MST technique, we can quantitatively describe the sample under investigation, divide the sample into physically motivated subsamples of the LSS elements with various threshold overdensities bounding them and characterize their morphology. In particular, this technique allows us to discriminate between filamentary and wall-like structure elements located presumably within low and high density regions and to estimate their parameters for the different threshold overdensities. The same technique allows us to extract sets of high density groups of galaxies and to measure some of their properties.

The analysis of wall-like condensations is most informative. Comparison of the observed characteristics of walls with theoretical expectations (DD99; DD02) demonstrates that the observed galaxy distribution is consistent with Gaussian initial perturbations and that the walls are the recently formed, partly relaxed Zel'dovich pancakes. The mean basic characteristics of the walls are consistent with those theoretically expected for the initial power spectrum measured by the CMB observations summarized, for example, in Spergel et al. (2003).

In this paper we also analyse the mass functions of structure elements selected for a variety of boundary threshold overdensities. We show that these functions are quite similar to the expectations of Zel'dovich theory, which generalizes the Press-Shechter formalism for any structure elements. In addition, the theory indicates that the interaction of large and small scale perturbations can be important for the formation of the observed LSS mass functions. Our analysis demonstrates that this interaction is actually seen in the influence of environment on the characteristics of groups of galaxies. This problem was also discussed in Einasto et al. (2003a,b).

This paper is organized as follows: in Sect. 2 we describe the sample of galaxies which we extracted from the SDSS DR1 and the method we have employed to correct for radial selection effects. In Sect. 3 we establish the general characteristics of the LSS. More detailed descriptions of the filamentary network and walls can be found in Sects. 4 and 5, respectively. In Sects. 6 and 7 we discuss the probable selected clusters of galaxies and the mass function of structure elements. We conclude with Sect. 8 where a summary and a short discussion of main results are presented.

\section{The data release 1 of the Sloan digital sky survey}

We use as our observational sample the SDSS DR1 (Abazajian et al. 2003), which is the first major public release of survey quality data from the SDSS (Fukugita et al. 1996; Gunn et al. 1998; York et al. 2000). The imaging data for the SDSS DR1 encompasses 2099 sq deg of sky. The DR1 also contains 186240 follow up spectra, which are available over $1360 \mathrm{sq}$ deg of the imaging data area. Galaxies are situated within two north fields, $N_{1}$ and $N_{2}$, and two south fields, $S_{1}$ and $S_{2}$. These regions are plotted in Fig. 1.

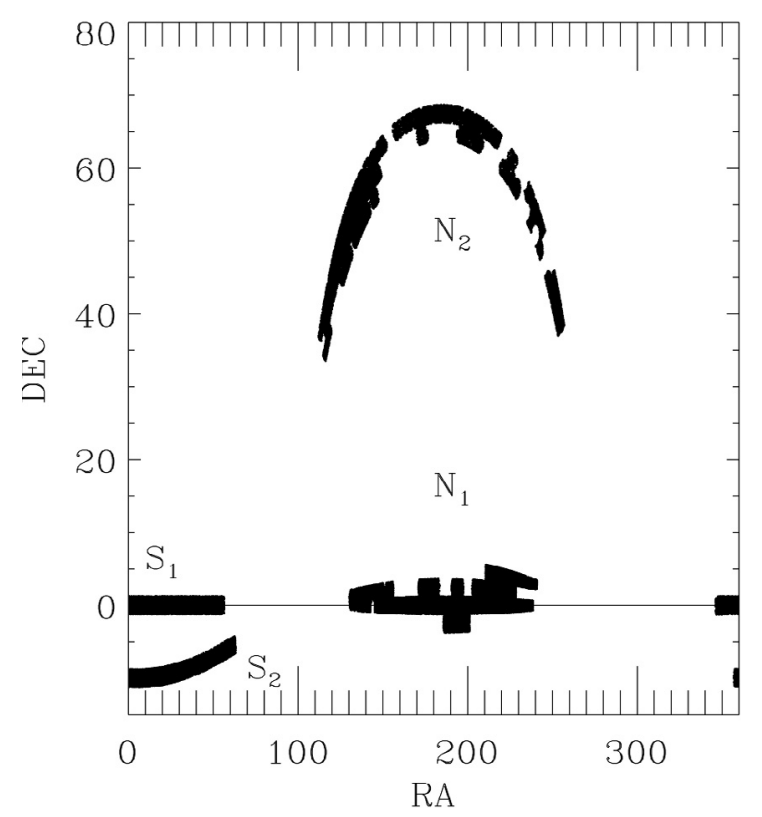

Fig. 1. Four regions of the DR1 sample on the sky.

We obtained our SDSS DR1 sample via the SDSS DR1 Spectro Query Server ${ }^{1}$. This is a web interface to the SDSS data archive server. We selected all objects identified as galaxies with a redshift confidence level of $\geq 95 \%$. No other constraints in the selection were made at this stage.

Our method for detecting LSS depends on having largely contiguous regions. Hence we have removed some regions and artifacts from the DR1 sample. The following are the RA and Dec areas we masked out from the original DR1 query before our analysis:

- $174^{\circ}<\mathrm{RA}<179^{\circ},-4.0^{\circ}<$ Dec $<-1.22^{\circ}$

- $159^{\circ}<\mathrm{RA}<163^{\circ}, 1.1^{\circ}<\operatorname{Dec}<4.0^{\circ}$

- $10^{\circ}<\mathrm{RA}<50^{\circ}, 10^{\circ}<\operatorname{Dec}<20^{\circ}$

- $300^{\circ}<\mathrm{RA}<355^{\circ},-12^{\circ}<\operatorname{Dec}<-4^{\circ}$

- $250^{\circ}<\mathrm{RA}<270^{\circ}, 52^{\circ}<\operatorname{Dec}<67^{\circ}$.

\subsection{Completeness of the survey and volume-limited samples}

Our analysis data set consists of both the SDSS main galaxy redshift sample (Strauss et al. 2002) and the SDSS Luminous Red Galaxy (LRG) redshift sample (Eisenstein et al. 2001). The SDSS main galaxy redshift sample is a flux-limited survey, with a faint-end limit of $r=17.77$ and an effective bright-end limit of $r \approx 15.0$ and an explicit $r$-band Petrosian half-light surface brightness limit of $\mu_{50} \leq 24.5 \mathrm{mag} \operatorname{arcsec}^{-2}$ (Strauss et al. 2002). Tests indicate that, within these limits, the SDSS targeting algorithm yields a set of main galaxy targets which is complete at the $99 \%$ level overall, degrading to about $95 \%$ for galaxies brighter than $r=15$, primarily due to deblending problems in the imaging catalog; about $6 \%$ of selected galaxies are not targeted spectroscopically due to the 55" fiber separation constraint (Strauss et al. 2002). Essentially all (99.9\%) of

\footnotetext{
${ }^{1}$ http://www.sdss.org/dr1
} 


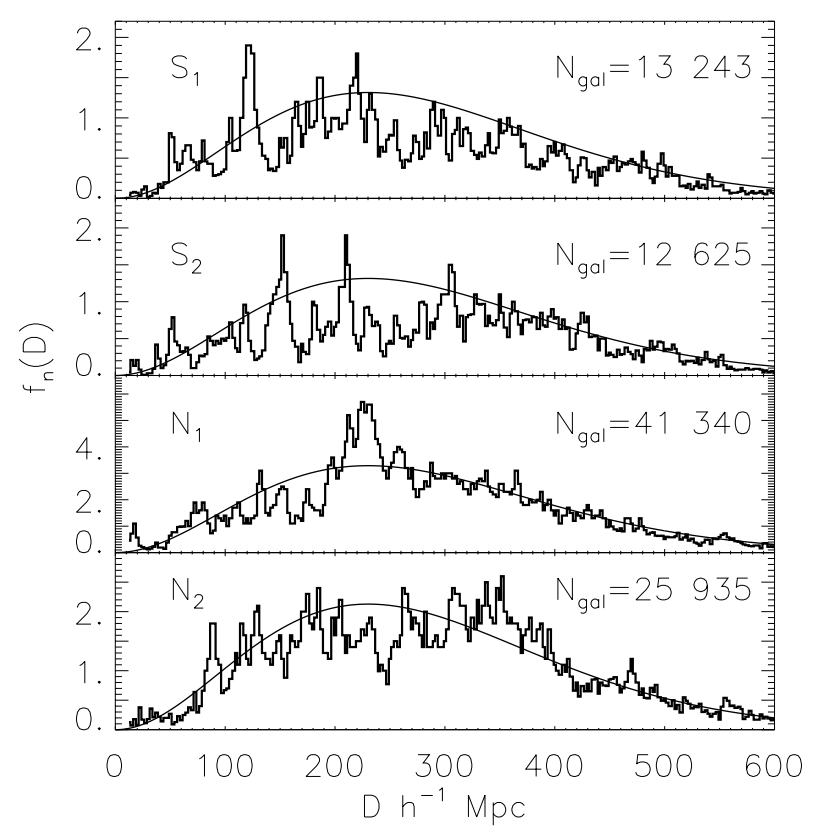

Fig. 2. The radial galaxies distributions in the four samples of the SDSS DR1. The selection function (1) is plotted by solid lines.

the targeted objects in the main galaxy sample yield a reliable redshift (Strauss et al. 2002). The inclusion of the full LRG redshift sample adds an additional $10-15 \%$ to our data set, mostly at $r>17.77$. As such, it is a useful but minor component of our full analysis set.

Therefore, to first order our analysis data set is essentially a flux-limited redshift survey with effectively both a faint and a bright-end magnitude limit. As such, it is difficult to create volume-limited samples which span a wide range of distances and which densely sample the galaxy distribution. One must trade off between these two competing goals, either choosing a deep-but-sparsely-sampled volume-limited data set or choosing a more densely sampled volume-limited sample that only covers a narrow range of distances. This can and has been done for the SDSS for a variety of studies, including those by Hoyle et al. (2002), Gómez et al. (2003), and Goto et al. (2003). For the analyses in the present paper, however, both depth and dense sampling are necessary in order to yield useful results. An illustrative example of this problem can be seen in the log$\log$ plot in Fig. 3 of Tegmark et al. (2003), where it is clear that we would need to discard substantially over $90 \%$ of the SDSS DR1 galaxies in order to get a sample depth out to a comoving distance of $400 h^{-1} \mathrm{Mpc}$.

\subsection{Correction for radial selection effects}

In Fig. 2 we plot the radial distributions of galaxies in all four samples. In Fig. 3 we plot the wedge diagram of the observed galaxy distribution for the sample $S_{1}$. As is clearly seen from these figures, at distances $D \geq 400 \mathrm{~h}^{-1} \mathrm{Mpc}$ this fraction of observed galaxies is strongly suppressed because of the radial selection effect. Note that this suppression is quite successfully fit by curves describing a selection function of the form

$f_{\text {gal }}(D) \propto D^{2} \exp \left[-\left(D / R_{\text {sel }}\right)^{3 / 2}\right], \quad R_{\text {sel }} \approx 190 h^{-1} \mathrm{Mpc}$,

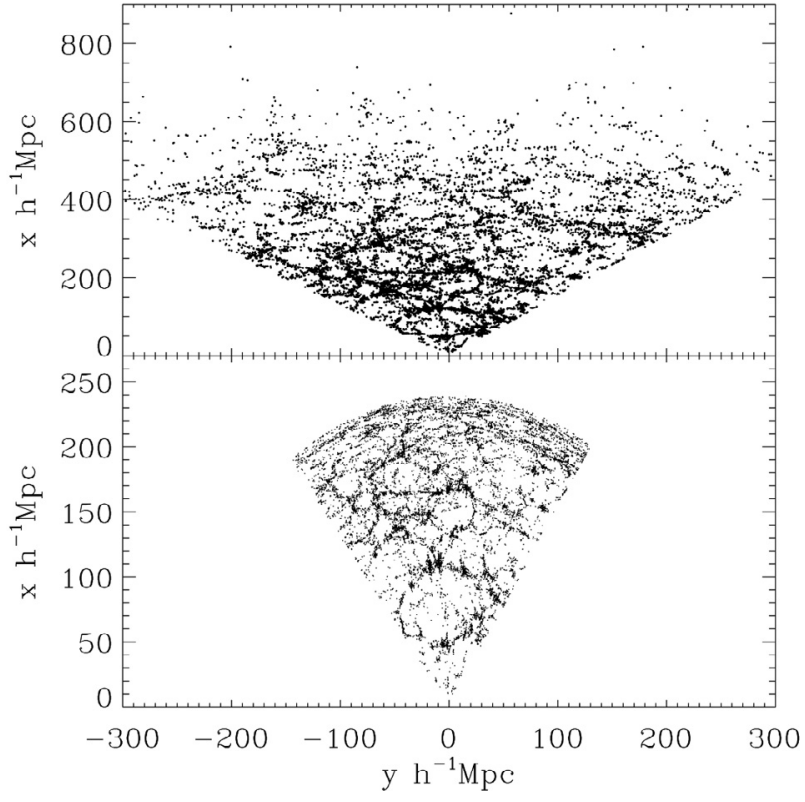

Fig. 3. The wedge diagram of galaxy distributions in the samples $S_{1}$ is real (top panel) and modified (bottom panel) radial coordinates.

where $D$ is a galaxy's radial distance and $R_{\text {sel }}$ is the selection scale (Baugh \& Efstathiou 1993). These fits are also plotted in Fig. 2.

In some applications, like when we want to correct a measure of the observed density to a measure of the true density, we would like to use Eq. (1) to correct for the radial selection effects after the fact. An example of such a case is calculating a group's or cluster's true richness based upon the observed number of galaxies it contains (Sects. 6 and 7).

In other applications, however, like in searching for groups or clusters in a magnitude-limited sample, we want to make a preemptive correction for the radial selection effects. For example, in a standard "friends-of-friends" percolation algorithm (e.g. Huchra \& Geller 1982), this is done by adjusting the linking length as a function of radial distance. Here, instead, we employ the rather novel approach of adjusting the radial distances themselves as introduced and discussed in Doroshkevich et al. (2001). Hence, instead of the measured radial distance, we use a modified radial distance, $D_{\mathrm{md}}$, where

$D_{\text {md }}^{3}=2 R_{\text {sel }}^{3}\left(1-\left[1+\left(D / R_{\text {sel }}\right)^{3 / 2}\right] \exp \left[-\left(D / R_{\text {sel }}\right)^{3 / 2}\right]\right)$.

The radial variations of the normalized number density of galaxies for all samples from Fig. 2 are plotted in Fig. 4. As is seen from this figure, the modified radial distances for the galaxies suppresses the very large-scale trends. On the other hand the relative positions of galaxies remain unchanged and the smaller scale random variations in the density are emphasized. The wedge diagram of the modified galaxy distribution for the sample $S_{1}$ is also plotted in Fig. 3 .

This correction does not change distances at $D \leq R_{\mathrm{sel}}$ and is more important for the more distant regions of our samples $\left(D \geq 350 h^{-1} \mathrm{Mpc}\right.$ ), which contain only $\sim 20 \%$ of all galaxies. Thus, in the following analyses, we apply this correction only to the separation of the high and low density regions in the deeper samples. Of course, it cannot restore the lost 


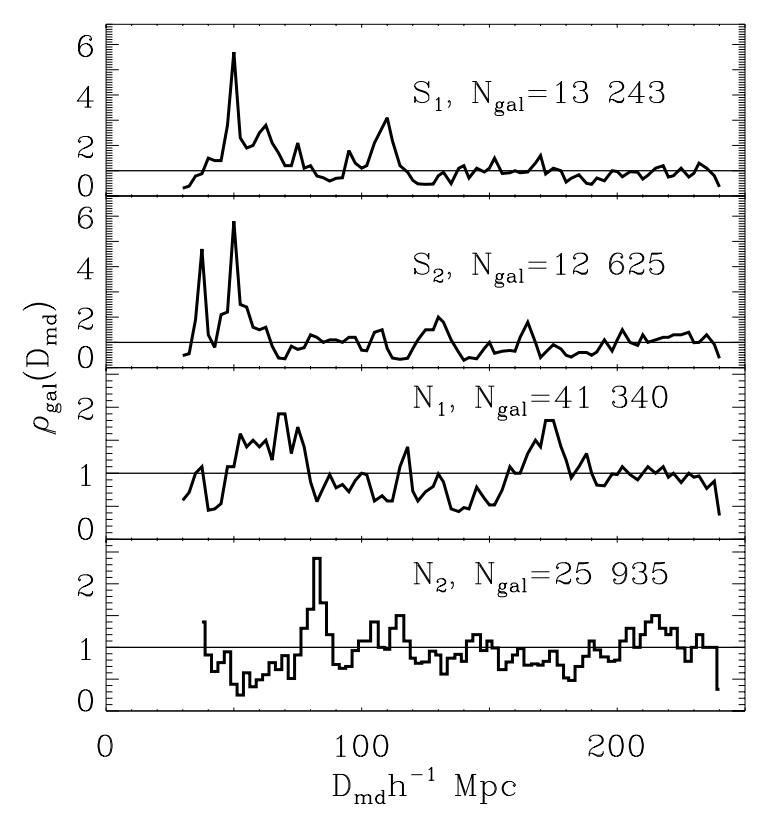

Fig. 4. The normalized mean galaxy density in the four modified samples of SDSS DR1.

information about the galaxy distribution in these regions, but it does help compensate for the strong drop in the observed galaxy density at these distances. It also allows one to apply the standard methods of investigation for the full catalogues with a depth of $600 \mathrm{~h}^{-1} \mathrm{Mpc}$.

\subsection{Samples of galaxies under investigation}

In our analysis here, we consider the four fields, plotted in Fig. 1, at the distance $D \leq 420 h^{-1} \mathrm{Mpc}$ with

$N_{\text {gal }}=79183,\left\langle n_{\text {gal }}\right\rangle \approx 10^{-2} \mathrm{Mpc}^{-3}$,

where $N_{\text {gal }}$ and $\left\langle n_{\text {gal }}\right\rangle$ are the total number of galaxies and the mean density of the samples. This sample contains $\approx 85 \%$ of all galaxies with moderate impact from selection effects. The numbers of galaxies in the separate fields are

- $N_{1}$, the northern sample (35 520 galaxies)

$-N_{2}$, the northern sample (21983 galaxies)

- $S_{1}$, the southern sample (11225 galaxies)

$-S_{2}$, the southern sample (10455 galaxies).

Both the depth and the selection scale of this survey are about $15 \%$ larger than those for the LCRS. In spite of the limited depth of the catalogue it allows us to separate rich samples of both filament-like and wall-like structure elements. For the mean separation of walls $\left(50-60 h^{-1} \mathrm{Mpc}\right)$ we can expect to observe 3-5 rich walls in each of four subsamples along many radial lines of sight. Both factors significantly increase the statistical representativeness of the measurements of the local characteristics of walls. However, the relative thinness of these slices $\left(\sim 2.5^{\circ}\right)$ can partly restrict the measured masses and sizes of richer walls and partly disrupts the joint network of the LSS (see discussion in Doroshkevich et al. 2001). However, for half of the galaxies located in the subsample $N_{1}$ this effect is attenuated.
For the expected length of filaments $\left(\sim 10-20 h^{-1} \mathrm{Mpc}\right)$ the possible impact of the finite size of our sample seems to be small. However, properties of the filaments are more sensitive to the incompleteness of the sample discussed in Sect. 2.1. This means that the analysis of the SDSS DR1 survey can provide important but limited information about quantitative characteristics of the LSS.

\section{General characteristics of observed large scale structure}

To characterize the general properties of the large scale spatial galaxy distribution we use the Minimal Spanning Tree (MST) technique applied both to directly observed samples of galaxies and to samples corrected for the selection effect.

\subsection{The MST technique}

The MST technique was first discussed by Barrow et al. (1985) and by van de Weygaert (1991). The MST is a construct from graph theory, originally introduced by Kruskal (1956) and Prim (1957), which has been widely applied in telecommunications and similar fields. It is a unique network associated with a given point sample and connects all points of the sample to a tree in a special and unique manner which minimizes the full length of the tree. Further definitions, examples, and applications of this approach are discussed in Barrow et al. (1985) and van de Weygaert (1991). More references to the mathematical results can also be found in van de Weygaert (1991).

One of earliest uses of MST approach in the study of largescale structure was that of Bhavsar \& Ling (1988), who successfully applied it to extract filamentary structures from the original CfA Redshift Survey. Its applications for the quantitative description of observed and simulated catalogues of galaxies were discussed in Demiański et al. (2000), Doroshkevich et al. (2000), Doroshkevich et al. (2001).

One of the most important features of the MST technique is generalization of the widely used "friends-of-friends" approach. It allows one to separate all LSS elements with a given linking length. In spite of the very complex shape of a typical LSS element, the linking length defines for each two points the local overdensity bounding this element with a relation familiar from "friends-of-friends" algorithms (Huchra \& Geller 1982):

$\delta_{\mathrm{thr}}=3 /\left[4 \pi\left\langle n_{\mathrm{gal}}\right\rangle r_{\mathrm{lnk}}^{3}\right]$

Further on it allows one to obtain characteristics of each element and of the sample of such elements forming the LSS with a given overdensity. Further discrimination can be performed for a given threshold richness of individual elements.

Here we will restrict our investigation to our results for the probability distribution function of the MST edge lengths $W_{\mathrm{MST}}(l)$ and to the morphological description of individual structure elements. The potential of the MST approach is not, however, exhausted by these applications. Examples of Minimal Spanning Tree are presented in van de Weygaert (1991) for simple models and in Doroshkevich et al. (2001) for one slice of the LCRS. 


\subsection{Wall-like and filamentary structure elements}

With the MST technique we can demonstrate that the majority of galaxies are concentrated within wall-like structures and filaments which connect walls to the joint random network of the cosmic web. The decomposition of the LSS into filaments and walls agrees with visual impressions and is suitable for purposes of discussion but, inevitably, this decomposition into two well-defined morphological subclasses is arbitrary. Indeed, structure evolution implies the continual transformation of sheet-like elements into filaments which are in turn integrated into richer walls. Richer filamentary-like elements are usually surrounded by the sheet-like halos and include a significant fraction of low mass high density clouds. In turn, wall-like elements incorporate a significant fraction of filaments. This means that the decomposition of walls, filaments, and clouds - and our consequent statistical analyses - depend upon the chosen threshold overdensity bounding the elements and vary with them. This means also that richer structure elements can be more correctly characterized by their degree of "filamentarity" and "wall-ness".

In spite of this, the observed galaxy distribution can be described as a set of one, two, and three dimensional Poisson-like distributions. Naturally, a one dimensional distribution is more typical for filaments while two and three dimensional ones are typical for walls and groups of galaxies, respectively. As was shown in van de Weygaert (1991) and Buryak \& Doroshkevich (1996), a Poissonian distribution of galaxies within the LSS elements successfully reproduces the observed 3D correlation function of galaxies.

These result show that the probability distribution function of MST edge lengths (PDF MST), $W_{\mathrm{MST}}(l)$, characterizes the geometry of the galaxy distribution. For $1 \mathrm{D}$ and 2D Poissonian distributions, typical for filaments and walls, $W_{\mathrm{MST}}(l)$ is described by the following exponential and Rayleigh functions,

$W_{\mathrm{MST}}(l)=W_{\mathrm{e}}(l)=\langle l\rangle^{-1} \exp (-l /\langle l\rangle)$,

$W_{\mathrm{MST}}(l)=W_{\mathrm{R}}(l)=2 l /\left\langle l^{2}\right\rangle \exp \left(-l^{2} /\left\langle l^{2}\right\rangle\right)$.

These PDFs remain valid for any 1D and 2D distributions when the galaxy separation is small as compared with the curvature of the lines and surfaces. Comparison of measured and expected PDFs MST allows one to demonstrate the existence of these two types of structure elements and to make approximate estimates of their richness. Let us recall, however, that for the galaxy groups embedded within filaments, 2D and 3D Poissonian distributions are observed and, so, in this case we cannot see a purely $1 \mathrm{D}$ distribution. For walls this effect is less important because it only distorts the 2D distribution typical for such LSS elements.

In Fig. 5 (top panel) we plot the $W_{\mathrm{MST}}\left(l_{\mathrm{MST}}\right)$ 's for the entire sample of 79183 galaxies situated at distances $D \leq$ $420 \mathrm{~h}^{-1} \mathrm{Mpc}$ where, as is seen from Fig. 2, the impact of the selection effect is still moderate. The error bars show the scatter of measurements for the four subsamples. For each sample, $N_{1}, N_{2}, S_{1}, S_{2}$, we have

$\left\langle l_{\mathrm{MST}}\right\rangle=2.5,2.6,2.3,2.0 h^{-1} \mathrm{Mpc}$.

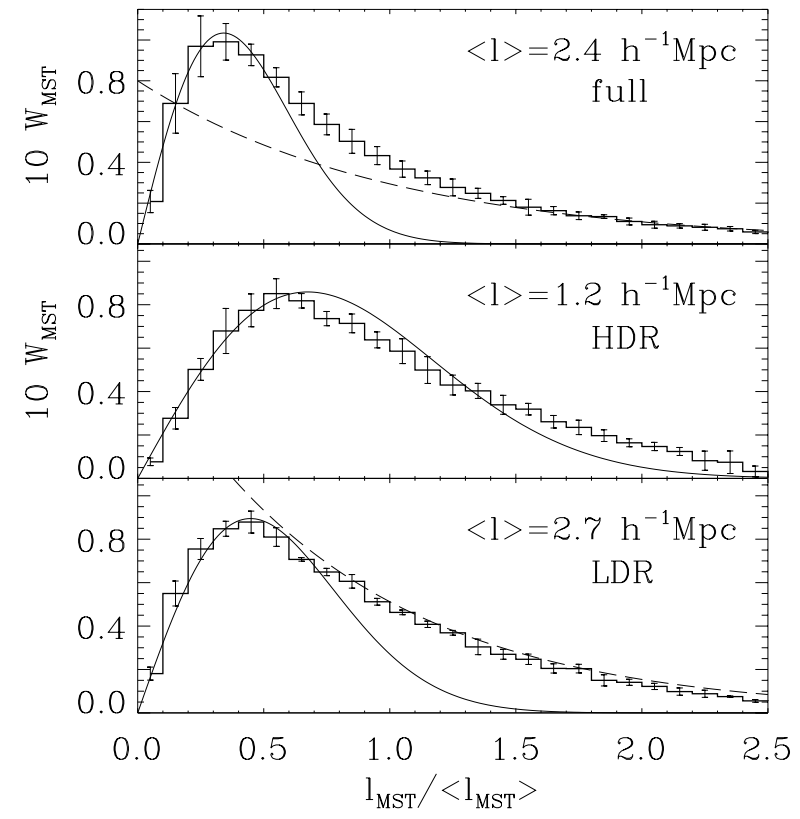

Fig. 5. PDFs of MST edge lengths in redshift space averaged over four samples are plotted for the full sample (top panel), HDRs (middle panel) and LDRs (bottom panel). Rayleigh and exponential fits are plotted by thin solid and dashed lines.

These variations demonstrate the differences in the sample properties (cosmic variance).

Notice in Fig. 5 that the $W_{\mathrm{MST}}\left(l_{\mathrm{MST}}\right)$ is well fit by a superposition of Rayleigh (at $l_{\mathrm{MST}} \leq\left\langle l_{\mathrm{MST}}\right\rangle, \sim 60 \%$ of galaxies) and exponential (at $l_{\mathrm{MST}} \geq\left\langle l_{\mathrm{MST}}\right\rangle, \sim 40 \%$ of galaxies) functions. This confirms results discussed in Doroshkevich et al. (2000, 2001) with respect to the high degree of galaxy concentration within the population of high density rich wall-like structures and less rich filaments. However, as was noted in the same papers, with this approach the approximate separation of wall-like and filamentary structure elements can be performed only statistically. This is because the high density part of the PDF described by the Rayleigh function includes high density clouds situated in both filaments and walls. The exponential part of the PDF is related mainly to the filamentary component.

\subsection{High and low density regions}

The methods for an approximate statistical decomposition of a sample into subsamples of wall-like structures and filaments were proposed and tested in our previous publications (Demiański et al. 2000; Doroshkevich et al. 2000, 2001). The first step is to make a rough discrimination between the high and low density regions (HDRs and LDRs).

Such discrimination can be easily performed for a given overdensity contour bounding the individual elements and a given threshold richness of them. Following Doroshkevich et al. (2001), in all four samples with $D \leq 420 h^{-1} \mathrm{Mpc}$, wall-like high density regions (HDRs) were identified with galaxy groups found for a threshold richness $N_{\text {thr }}=40$ and a threshold overdensity contour bounding them equal to the mean density, $\delta_{\text {thr }}=1$. These samples, $N_{1}, N_{2}, S_{1}, S_{2}$, of HDRs 
contain $49 \%, 47 \%, 51 \%$ and $47 \%$ of all galaxies. The samples of low density regions (LDRs), which are occupied mainly by filaments and poor groups of galaxies, are complementary to the HDRs in that the LDRs are simply the leftovers from the original total samples after the HDRs have been removed.

The matter fraction incorporated within the HDRs (and, complementarily, within LDRs) depend strongly upon the values of the parameters $N_{\mathrm{thr}}$ and $\delta_{\mathrm{thr}}$; for instance, the matter fraction contained within HDRs increases for smaller $N_{\mathrm{thr}}$. As a rough estimate of the maximum fraction of galaxies incorporated within HDRs in the DR1 sample we can take the value $\sim 60 \%$ obtained in the previous section. For the values of the parameters utilized, the properties of HDRs are quite similar to those expected for walls which verifies this choice of values. However, the identification of these so defined HDRs and LDRs with "walls" and "filaments" is approximate and below the same methods of decomposition will be applied to the selected HDRs and LDRs themselves to reveal their own internal high density components and to link them with an environment.

In Fig. 5 (middle panel) the $W_{\mathrm{MST}}(l)$ plotted for the HDRs is very similar to a Rayleigh function, thus confirming with this criterion the sheet-like nature of the observed galaxy distribution within the HDRs. As before, the error bars show the scatter of measurements for the four subsamples. For $90 \%$ of objects we have

$W_{\mathrm{HDR}}=(1 \pm 0.18) W_{\mathrm{R}}, \quad l_{\mathrm{MST}} \leq 1.65\left\langle l_{\mathrm{MST}}\right\rangle$,

where $W_{\mathrm{R}}$ is the Rayleigh function (5). A larger difference between observed and expected PDFs for larger $l_{\mathrm{MST}}$ indicates that the selected sample of HDRs includes some fraction of objects, $\sim 10 \%$, which can be related to the filamentary component with the exponential PDF.

For the LDRs, the $W_{\mathrm{MST}}(l)$ is plotted in Fig. 5 (bottom panel). For small edge lengths, $l \leq\left\langle l_{\mathrm{MST}}\right\rangle$, it also fits well to a Rayleigh function indicating that $\sim 60 \%$ of LDR galaxies are concentrated within less massive 3D (elliptical) and 2D (sheetlike) high density clouds. This result confirms the strong disruption of filaments into a system of clouds. For larger edge lengths, however, the LDR $W_{\mathrm{MST}}(l)$ appears to be closer to an exponential function, indicating that according to this criterion the spatial distribution of the remaining $\sim 40 \%$ of LDR galaxies is similar to a 1D Poissonian one which is typical for filamentary structures.

The mean edge lengths, $\left\langle l_{\mathrm{MST}}\right\rangle$, found for HDRs and LDRs in samples $N_{1}, N_{2}, S_{1}, S_{2}$ are

$\left\langle l_{\mathrm{MST}}\right\rangle=1.2,1.3,1.2,1.3 h^{-1} \mathrm{Mpc}$,

$\left\langle l_{\mathrm{MST}}\right\rangle=2.8,2.7,2.6,2.8 h^{-1} \mathrm{Mpc}$.

These values differ by about a factor of two from each other, indicating that, as is seen from (4), the difference in the mean density within HDRs and LDRs elements is about an order of magnitude. Of course, the volume averaged density of LDRs is still less.

\subsection{Morphology of the structure elements}

Within so defined HDRs and LDRs themselves we can extract with the MST technique subsamples of structure elements for various threshold overdensities. We can then suitably characterize the morphology of each structure element by comparing the sum of all edge lengths within its full tree, $L_{\text {sum }}$, with the sum of all edge lengths within the tree's trunk, $L_{\mathrm{tr}}$, which is the longest path that can be traced along the tree without re-tracing any steps. The ratio of these lengths

$\epsilon=L_{\mathrm{tr}} / L_{\mathrm{sum}}$

suitably characterizes the morphology of the LSS elements.

For filaments, we can expect that the lengths of the full tree and of the trunk are similar to each other, $\epsilon \sim 1$, whereas for clouds and walls these lengths are certainly very different and $\epsilon \leq 1$. This approach takes into account the internal structure of each element rather than the shape of the isodensity contour bounding it, and in this respect it is complementary to the Minkowski Functional technique (e.g., Schmalzing et al. 1999; Sheth et al. 2002).

However, even this method cannot discriminate between the wall-like and 3D (elliptical) clouds and those rich filaments having many long branches for which again $\epsilon \leq 1$. This means that both the PDF of this ratio, $W(\epsilon)$, and the corresponding mass fraction, $f_{m}(\epsilon)$, are continuous functions and the morphology of structure elements can be more suitably characterized by the degree of filamentarity and "wall-ness". This also means that we can only hope to distinguish statistical differences between the morphologies of structure elements in HDRs and the morphologies of structure elements in LDRs.

The decomposition of HDRs and LDRs was performed for two threshold linking lengths, $r_{\text {lnk }}=2$ and $2.4 h^{-1} \mathrm{Mpc}$ for HDRs, and $r_{\text {lnk }}=3.2$ and $3.6 h^{-1} \mathrm{Mpc}$ for LDRs. These values are larger than the mean edge lengths and characterize the LSS elements with intermediate richness when the measured difference between the walls and filaments is maximal. As was noted above, for lower linking lengths this method characterizes mainly the internal structure of the LSS elements while for large linking lengths filaments percolate and form the joint network with again $\epsilon \ll 1$.

The distribution functions of the ratio, $W(\epsilon)$, are found to be close to Gaussian with $\langle\epsilon\rangle \approx 0.5$ and 0.70 for HDRs and LDRs, respectively. The mass fractions, $f_{\mathrm{m}}(\epsilon)$, plotted in Fig. 6 for the same linking lengths are shifted to the left (for HDRs) and to the right (for LDRs) with respect to the middle point. Differences between these fractions found for smaller and larger linking lengths illustrate the impact of the percolation process and disruption of the LSS elements.

These results verify the objective nature of the differences in the structure morphologies in HDRs and LDRs.

\section{Statistical characteristics of filaments}

Theoretical characteristics of the LSS elements relate to the dominant dark matter (DM) component while the observed galaxy distribution relates to the luminous matter which represent only $\sim 3-5 \%$ of the mean density of the Universe. The spatial distribution of dark and luminous matter is strongly biased. None the less, some observed characteristics of filamentary components of the LSS can be compared with both 


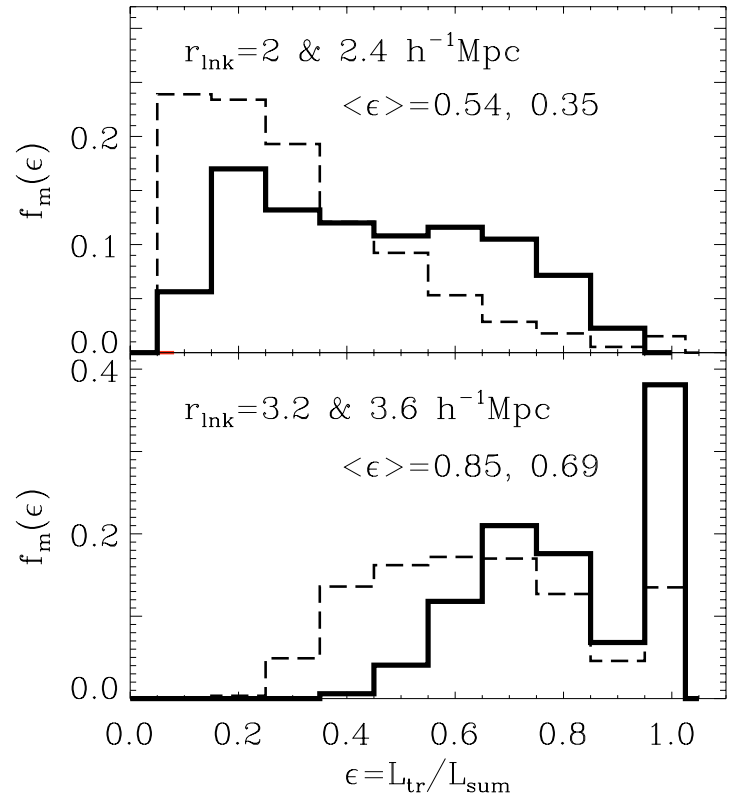

Fig. 6. Variations of the mass fraction, $f_{m}(\epsilon)$, vs. $\epsilon=L_{\text {tr }} / L_{\text {sum }}$ for the structure elements selected within HDRs (top panel, solid and dashed lines) and within LDRs (bottom panel, solid and dashed lines).

available theoretical expectations and characteristics obtained for simulated DM distributions.

The most interesting ones are the PDF of the linear density of filaments measured as the mass or number of objects per unit length of filament, $\Sigma_{\text {fil }}$. The other is the mean surface density of filaments $\sigma_{\text {fil }}$, defined as the mean number of filaments intersecting a unit area of arbitrary orientation. Both characteristics depend upon the threshold linking length, $r_{\text {lnk }}$, used for the filament selection and upon the threshold richness of filaments. However, both characteristics are independent of the small scale clustering of matter within filaments.

Comparison of these characteristics of filaments for observed and simulated catalogues allows one to test the cosmological model used. However, the connection of quantitative characteristics of filaments with the initial power spectrum is complex and these measurements cannot yet be used for estimates of the power spectrum.

\subsection{Linear density of filaments}

As was found in DD99 and DD02, for the CDM-like initial power spectrum the PDF of the filament linear number density can be written as follows:

$N_{\text {fil }} \mathrm{d} \Sigma_{\text {fil }} \approx \frac{1.5}{\left\langle\Sigma_{\text {fil }}\right\rangle} \exp \left(-\sqrt{3 \Sigma_{\text {fil }} /\left\langle\Sigma_{\text {fil }}\right\rangle}\right) \mathrm{d} \Sigma_{\text {fil }}$.

However, in both simulated and observed catalogues the filament linear number density is restricted by the linking length used for the selection of filaments, $\Sigma_{\text {fil }} \geq 1 / r_{\text {lnk }}$, which introduces an artificial cutoff in the PDF (11). Hence, we will fit the measured PDF to the relation

$N_{\text {fil }} \approx a_{0} \operatorname{erf}^{4}\left[a_{1}\left(x-x_{0}\right)\right] \exp \left[-\sqrt{a_{2}\left(x-x_{0}\right)}\right]$

where for high resolution simulations $x=\Sigma_{\text {fil }} /\left\langle\Sigma_{\text {fil }}\right\rangle, x_{0} \approx$ $0.35, a_{1} \approx 2-2.5$ and $a_{2} \approx 30-40$ (DD02) and $\left\langle\Sigma_{\text {fil }}\right\rangle \sim 3 / r_{\text {lnk }}$.

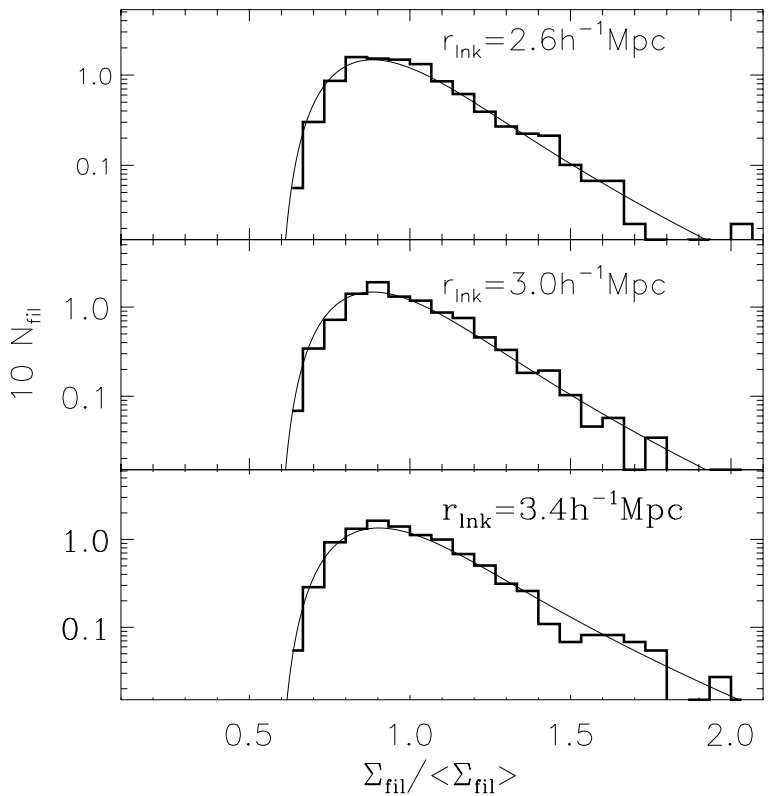

Fig. 7. Distribution function, $N_{\text {fil }}$, for the linear density of "galaxies" along a tree for filaments selected in LDRs of the mock catalogues with three linking lengths. Fits (11) are plotted by solid lines.

The cutoff of the PDF (12) at $x \sim x_{0}$ described by the first term reflects only the limitations of the measurement. The impact of this term is small at $\Sigma_{\text {fil }} \geq\left\langle\Sigma_{\text {fil }}\right\rangle$ where the measured PDF becomes similar to the expected one (11).

These results can be compared with the ones obtained for for the DR1 and the mock catalogue (Cole et al. 1998), which allows one to estimate the impact of the selection effect for the measured linear density of filaments. The mock catalogues used are based on the high resolution DM simulation for the spatially flat $\Lambda$ CDM cosmological model with $\Omega_{\Lambda}=0.7, \Omega_{\mathrm{m}}=$ 0.3 transformed to the real geometry and depth of the $2 \mathrm{dF}$ and the SDSS surveys with a realistic selection function. In both the cases, the linear density of objects was measured by the ratio of the number of points and the length of the MST for each of the filaments of the sample.

For the mock catalogue the PDFs of the linear density of filaments are plotted in Fig. 7 for three linking length (with $\delta_{\text {thr }} \approx 1,0.7$ and 0.5 ). These PDFs are well fit by expression (12) for parameters $x_{0} \approx 0.6, a_{1} \approx 2.5, a_{2} \approx 80-90$. For all linking lengths we get $\left\langle\Sigma_{\text {fil }}\right\rangle r_{\text {lnk }}=2.25 \pm 0.04$. For the observed DR1 catalogue the same PDFs are plotted in Fig. 8 also for three linking length (with $\delta_{\text {thr }} \approx 50,5$ and 0.55 ). They are well fit by the same expression (12) for parameters $x_{0} \approx 0.5, a_{1} \approx$ $2.5, a_{2} \approx 70-80$. For this sample we get $\left\langle\Sigma_{\mathrm{fil}}\right\rangle r_{\mathrm{lnk}}=2.2 \pm 01$.

These results show that, in all the cases, the measured PDFs are well fit by the same expression (12) which coincides with the theoretically expected one (11) at $\Sigma_{\text {fil }} \geq\left\langle\Sigma_{\text {fil }}\right\rangle$. The mean linear density, $\left\langle\Sigma_{\mathrm{fil}}\right\rangle$, clearly depends upon the linking length used for filament selection. The selection effect increases the product $\left\langle\Sigma_{\text {fil }}\right\rangle r_{\text {lnk }}$ by $\sim 1.5$ times as compared with results obtained for the DM simulations.

These results show that the observed galaxy distribution nicely represents the expected and simulated ones. The results 


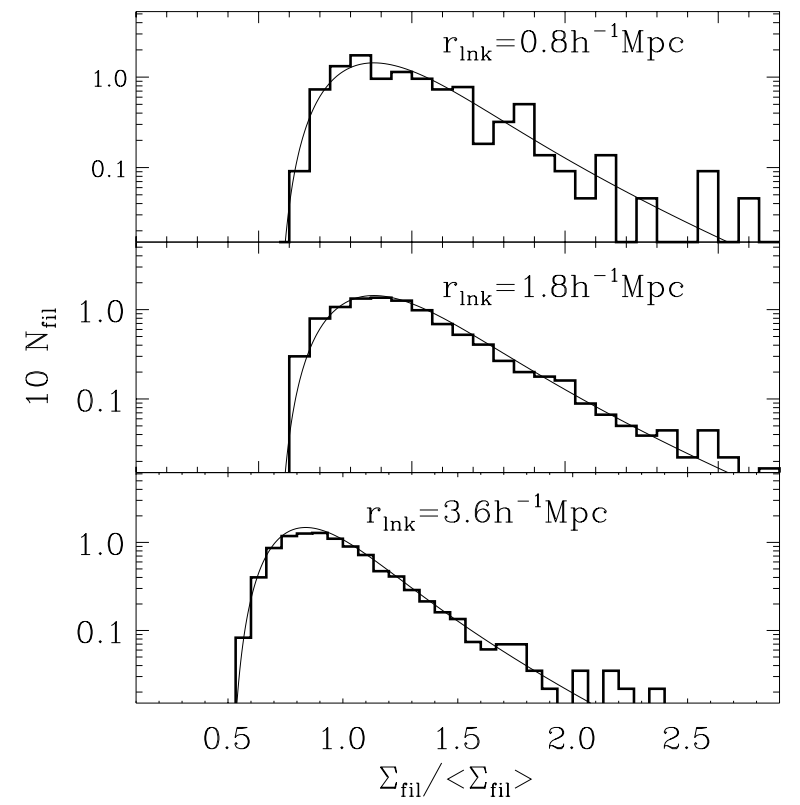

Fig. 8. Distribution function, $N_{\text {fil }}$, for the linear density of galaxies along a tree for filaments selected in LDRs of the DR1 with three linking lengths. Fits (11) are plotted by solid lines.

also indicate that the general properties of filaments are consistent with a CDM-like initial power spectrum.

\subsection{Typical size of the filamentary network}

Due to the complex shape of the network of filaments spanning the LDRs any definition of the typical size of a network cell is merely convenient. As was discussed in Doroshkevich et al. (2001), two definitions seem to be the most objective. One is the mean free path between filaments along a random straight line. The other is the mean distance between branch points of the tree along the trunk of selected filaments. The second definition tends to yield cell sizes that are typically a factor of 1.5 smaller than those yielded by the mean-free-path definition.

Theoretical estimates of this size are uncertain because it strongly depends upon the sample of selected filaments (DD02). This means that this characteristic strongly depends upon the catalogue used. Moreover, filaments are connected to the network only for larger linking lengths; thus the typical measured cell size depends also upon the threshold linking length used. Hence, for the LCRS the mean free path between filaments with a variety of richness was estimated in Doroshkevich et al. (2001) as $\sim 13-30 h^{-1} \mathrm{Mpc}$. The mean distance between branch points of the tree along the trunk was estimated as $\approx 10 h^{-1} \mathrm{Mpc}$ and it rapidly increases with the linking length used owing to the progressive percolation of filaments and the formation of the joint LSS network.

Here with a richer sample of filaments we can also estimate the PDFs of the cell sizes measured by the distance between branch points of the tree along the trunk. These PDFs, $N\left(l_{\mathrm{br}}\right)$, are plotted in Fig. 9 for two linking lengths, $r_{\text {lnk }}=1.8$ and 3.6 $h^{-1} \mathrm{Mpc}$, which correspond to the threshold overdensities

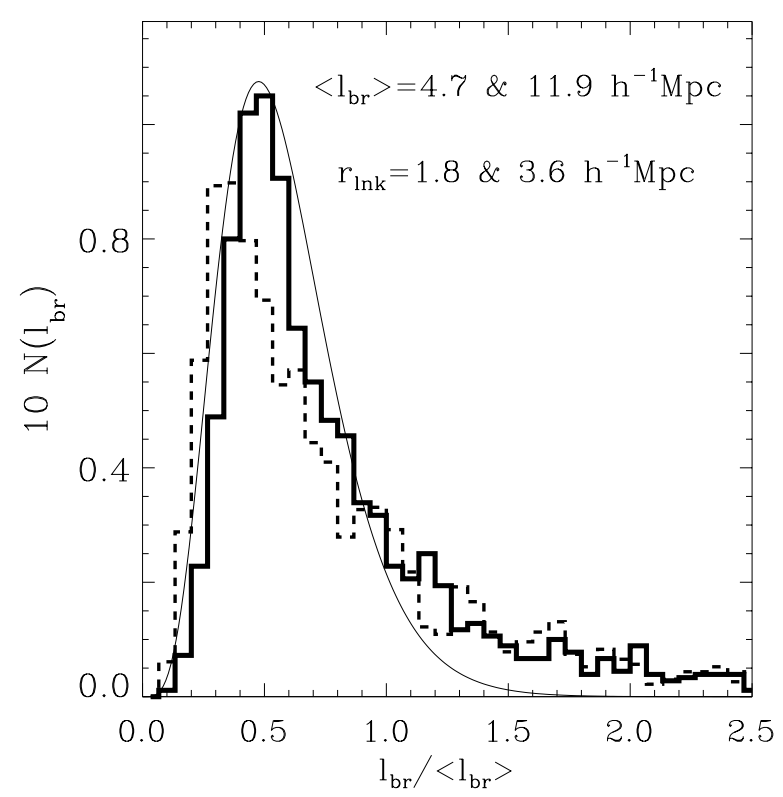

Fig. 9. Distribution functions, $N$, for the distance between branch points along a trunk for filaments selected in LDRs (thick solid and dashed lines). Fit (13) is plotted by thin solid line.

$\delta_{\text {thr }}=0.66$ and 0.5 . These PDFs are roughly fit by the expression,

$N\left(l_{\mathrm{br}}\right) \approx 270 x^{4.5} \exp (-9.1 x), \quad x=l_{\mathrm{br}} /\left\langle l_{\mathrm{br}}\right\rangle$.

The measured mean distance between branch points,

$\left\langle l_{\mathrm{br}}\right\rangle \approx 4.7$ and $11.9 h^{-1} \mathrm{Mpc}$,

are close to those obtained in Doroshkevich et al. (2001) and Doroshkevich et al. (1996) and those cited above. For smaller $r_{\text {lnk }}$ this estimate is decreased because of the domination of short filaments which are not yet connected to the network.

\section{Parameters of the wall-like structure elements}

The statistical characteristics of observed walls were first measured using the LCRS and DURS (Doroshkevich et al. 2000, 2001). The rich sample of walls extracted from the SDSS DR1, however, permits more refined estimates of these characteristics. As was discussed in Sect. 3.2, walls dominate the HDRs, and thus these subsamples of galaxies can be used to estimate the wall properties.

The statistical characteristics of walls depend upon the initial power spectrum and the cosmological model. Using the Zel'dovich theory of gravitational instability (Zel'dovich 1970; Shandarin \& Zeldovich 1989) the formation of walls can be described as the presumably one dimensional collapse of the matter which is driven by the initial velocity field. This means that the observed characteristics of walls can be expressed through the amplitude and correlation function of this field in the same manner as, for example, in the Press - Schechter formalism (see, e.g., Loeb \& Barkana 2001). For a CDM-like initial power spectrum and Gaussian perturbations, the main characteristics of walls were determined in DD99 and DD02. 
Measurement of the wall characteristics was discussed in Demiański et al. (2000) so here we will only briefly reproduce the main definitions. It is important that these characteristics can be measured independently in radial and transverse directions, which reveals the strong influence of the velocity dispersion within walls on other wall characteristics.

\subsection{Main wall characteristics}

The main characteristic of walls is their mean dimensionless surface density, $\left\langle q_{\mathrm{w}}\right\rangle$, measured by the number of galaxies per $\mathrm{Mpc}^{2}$ and normalized by the mean density of galaxies multiplied by a coherent length of the initial velocity field (DD99; DD02)

$l_{\mathrm{v}} \approx 33 h^{-1} \mathrm{Mpc}(0.2 / \Gamma), \quad \Gamma=\Omega_{\mathrm{m}} h$,

where $\Omega_{\mathrm{m}}$ is the mean matter density of the Universe. For Gaussian initial perturbations and the CDM-like power spectrum, the expected PDF of the surface density is

$N_{\mathrm{th}}\left(q_{\mathrm{w}}\right)=\frac{1}{\sqrt{2 \pi}} \frac{1}{\tau_{\mathrm{m}} \sqrt{q_{\mathrm{w}}}} \exp \left(-\frac{q_{\mathrm{w}}}{8 \tau_{\mathrm{m}}^{2}}\right) \operatorname{erf}\left(\sqrt{\frac{q_{\mathrm{w}}}{8 \tau_{\mathrm{m}}^{2}}}\right)$,

$\left\langle q_{\mathrm{w}}\right\rangle=\left\langle q_{\mathrm{th}}\right\rangle=8(0.5+1 / \pi) \tau_{\mathrm{m}}^{2} \approx 6.55 \tau_{\mathrm{m}}^{2}$.

This relation links the mean surface density of walls with the dimensionless amplitude of perturbations, $\tau_{\mathrm{m}}$,

$\tau_{\mathrm{m}}=\sqrt{\left\langle q_{\mathrm{w}}\right\rangle / 6.55}$

As is seen from (14-16), both the measured dimensionless surface density, $q_{\mathrm{w}}$, and the amplitude, $\tau_{\mathrm{m}}$, depend upon $\Gamma$, namely, $q_{\mathrm{w}} \propto \Gamma, \tau_{\mathrm{m}} \propto \sqrt{\Gamma}$. For the flat $\Lambda \mathrm{CDM}$ cosmological model with $\Omega_{\mathrm{m}}=0.3$ and for CDM-like power spectrum

$\tau_{\mathrm{m}} \approx 0.236 \sigma_{8}$

These measurements of amplitude can be compared with those obtained by other methods (DD02, Sect. 8.2).

Other important characteristics of walls are the mean velocity dispersion of galaxies within walls, $\left\langle w_{\mathrm{w}}\right\rangle$, the mean separation between walls, $\left\langle D_{\text {sep }}\right\rangle$, the mean overdensity, $\langle\delta\rangle$, and the mean thickness of walls, $\langle h\rangle$. The mean velocity dispersion of galaxies, $\left\langle w_{\mathrm{w}}\right\rangle$, can be measured in the radial direction only whereas other wall characteristics can be measured both radially and along transverse arcs. Comparison of the wall thickness and the overdensity, $\langle h\rangle$ and $\langle\delta\rangle$, measured in transverse (t) and radial (r) directions, illustrates the influence of the velocity dispersion of galaxies on the observed wall thickness.

In redshift space the radial velocity dispersion of galaxies within a wall, $w_{\mathrm{w}}$, is linked with the radial thickness of the wall (Demiański et al. 2000):

$h_{\mathrm{r}}=\sqrt{12} H_{0}^{-1} w_{\mathrm{w}}$.

Here the factor 12 normalizes the wall thickness to that of a homogeneous slab with the same surface density of galaxies.

For a relaxed, gravitationally confined wall, the velocity dispersion is equal to the depth of the wall potential well.
This depth is expressed through the wall overdensity and surface density as follows:

$w_{\mathrm{w}}^{2}=\frac{\pi G \mu^{2}}{\langle\rho\rangle \delta} \Theta_{\Phi}=\frac{3}{8} \frac{\Omega_{\mathrm{m}}}{\delta}\left(H_{0} l_{v} q_{\mathrm{w}}\right)^{2} \Theta_{\Phi}$.

Here $\mu=\langle\rho\rangle l_{v} q_{\mathrm{w}}$ is the mass surface density of the wall and the unknown factor $\Theta_{\Phi} \sim 1$ describes the possible nonhomogeneity of the matter distribution across the wall. Unfortunately, for these estimates we can only use the velocity dispersion and overdensity measured for radial and transverse directions, respectively. Hence, the final result cannot be averaged over the samples of walls.

\subsection{Measurement of the wall characteristics}

The characteristics of the walls can be measured with the two parameter core-sampling approach (Doroshkevich et al. 1996) applied to the subsample of galaxies selected within HDRs. With this method, all galaxies of the sample are distributed within a set of radial cores with a given angular size, $\theta_{\mathrm{c}}$, or within a set of cylindrical cores oriented along arcs of right ascension with a size $d_{\mathrm{c}}$. All galaxies are projected on the core axis and collected in a set of one-dimensional clusters with a linking length, $l_{\text {link}}$. The one-dimensional clusters with richnesses greater than some threshold richness, $N_{\min }$, are then used as the required sample of walls within a sampling core.

Both the random intersection of core and walls and the nonhomogeneous galaxy distribution within walls lead to significant random scatter of measured wall characteristics. The influence of these factors cannot be eliminated, but it can be minimized for an optimal range of parameters $\theta_{\mathrm{c}}, d_{\mathrm{c}}, l_{\text {link }}$ and $N_{\text {min }}$. Results discussed below are averaged over the optimal range of these parameters.

For the measurement of wall characteristics in the radial direction four samples of HDRs galaxies were used in each field of the DR1 catalogue. One of these samples was selected as was discussed in Sect. 3.3, three other samples were selected from the catalogues already corrected for radial selection effects (Sect. 2.1) with the same threshold overdensity $\delta_{\text {thr }}=1$ and for HDRs containing $\sim 43 \%, 50 \%$, and $56 \%$ of all galaxies in the field. In all the cases, the wall parameters were measured in real space for the selected samples of the HDRs. The mean wall properties were averaged over four radial core sizes $\left(\theta_{\mathrm{c}}=2^{\circ}, 2.25^{\circ}, 2.5^{\circ}\right.$ and $\left.2.75^{\circ}\right)$ and for six core-sampling linking lengths $\left(2 h^{-1} \mathrm{Mpc} \leq l_{\text {link }} \leq 4.5 h^{-1} \mathrm{Mpc}\right)$. Final averaging was performed over all sixteen samples and over all $\theta_{\mathrm{c}}$ and $l_{\text {link }}$.

Due to the complex shape of the fields $S_{2}$ and $N_{2}$, the measurements of wall characteristics in the transverse direction were performed for the fields $S_{1}$ and $N_{1}$ only. The mean wall properties were averaged over four core diameters $\left(d_{\mathrm{c}}=\right.$ 6.0, 6.5, 7.0, and 7.5 $\mathrm{h}^{-1} \mathrm{Mpc}$ ) and five core-sampling linking lengths $\left(2 h^{-1} \mathrm{Mpc} \leq l_{\text {link }} \leq 4 h^{-1} \mathrm{Mpc}\right)$.

\subsection{Measured characteristics of walls}

The mean radial and tranverse wall properties for all fields are listed separately in Table 1. Characteristics obtained by averaging over all samples are compared with those from the DURS 
Table 1. Wall properties in observed and simulated catalogues.

\begin{tabular}{|c|c|c|c|c|c|c|c|c|c|}
\hline Sample & $N_{\text {gal }}$ & $\left\langle q_{w}\right\rangle / \Gamma$ & $\tau_{m} / \sqrt{\Gamma}$ & $\left\langle\delta_{r}\right\rangle$ & $\begin{array}{c}\left\langle\delta_{t}\right\rangle \\
h^{-1} \mathrm{Mpc}\end{array}$ & $\begin{array}{c}\left\langle h_{r}\right\rangle \\
h^{-1} \mathrm{Mpc}\end{array}$ & $\begin{array}{c}\left\langle h_{t}\right\rangle \\
\mathrm{km} \mathrm{s}^{-1}\end{array}$ & $\begin{array}{c}\left\langle w_{w}\right\rangle \\
h^{-1} \mathrm{Mpc}\end{array}$ & $\left\langle D_{\text {sep }}\right\rangle$ \\
\hline \multicolumn{10}{|c|}{ Radial cores } \\
\hline N1 & 41217 & $2.01 \pm 0.21$ & $0.55 \pm 0.03$ & 1.3 & - & $10.2 \pm 1.9$ & - & $295 \pm 55$ & $69 \pm 14$ \\
\hline $\mathrm{N} 2$ & 25935 & $1.61 \pm 0.16$ & $0.49 \pm 0.03$ & 1.1 & - & $10.5 \pm 2.0$ & - & $303 \pm 57$ & $78 \pm 17$ \\
\hline S1 & 13215 & $2.21 \pm 0.19$ & $0.58 \pm 0.03$ & 1.4 & - & $10.5 \pm 1.7$ & - & $303 \pm 50$ & $67 \pm 14$ \\
\hline S2 & 12585 & $1.79 \pm 0.33$ & $0.51 \pm 0.04$ & 1.3 & - & $9.0 \pm 1.8$ & - & $260 \pm 50$ & $83 \pm 20$ \\
\hline \multicolumn{10}{|c|}{ Transverse cores for the SDSS DR1 } \\
\hline N1 & 16883 & $2.47 \pm 0.51$ & $0.61 \pm 0.07$ & - & 3.9 & - & $4.3 \pm 0.8$ & - & $65 \pm 11$ \\
\hline S1 & 13215 & $2.29 \pm 0.64$ & $0.58 \pm 0.08$ & - & 3.9 & - & $4.0 \pm 0.8$ & - & $58 \pm 11$ \\
\hline \multicolumn{10}{|c|}{ Observed samples } \\
\hline SDSS (radial) & 92952 & $1.91 \pm 0.32$ & $0.53 \pm 0.05$ & 1.3 & - & $10.1 \pm 2.0$ & - & $291 \pm 56$ & $74 \pm 17$ \\
\hline SDSS (transverse) & 29311 & $2.42 \pm 0.67$ & $0.60 \pm 0.08$ & - & 3.5 & - & $4.9 \pm 1.3$ & - & $64 \pm 14$ \\
\hline LCRS & 16756 & $2.51 \pm 0.9$ & $0.62 \pm 0.10$ & 3.0 & 7.4 & $8.6 \pm 0.8$ & $2.8 \pm 0.7$ & $247 \pm 48$ & $60 \pm 10$ \\
\hline DURS & 2500 & $2.23 \pm 0.6$ & $0.58 \pm 0.08$ & 1.7 & 6.5 & $9.7 \pm 1.8$ & $4.9 \pm 1.2$ & $280 \pm 52$ & $44 \pm 10$ \\
\hline \multicolumn{10}{|c|}{ Mock catalogues in real and redshift spaces for the model with $\Gamma=0.2$} \\
\hline Redshift & 98828 & $2.7 \pm 0.5$ & $0.63 \pm 0.06$ & 1.8 & 3.8 & $11.8 \pm 2.1$ & $6.5 \pm 1.4$ & $338 \pm 65$ & $50 \pm 10$ \\
\hline Real & 98828 & $2.1 \pm 0.4$ & $0.57 \pm 0.06$ & 4.3 & 4.6 & $4.8 \pm 1.0$ & $4.2 \pm 1.0$ & $305 \pm 47$ & $50 \pm 10$ \\
\hline \multicolumn{10}{|c|}{ DM catalogue in real space for the model with $\Gamma=0.2$} \\
\hline Real & $7.1 \cdot 10^{6}$ & $2.5 \pm 0.4$ & $0.63 \pm 0.04$ & 2.7 & & $4.9 \pm 0.5$ & & $245 \pm 30$ & $52 \pm 5$ \\
\hline
\end{tabular}

and LCRS and with those from mock catalogues simulating the SDSS EDR (Cole et al. 1998). Both DM simulation and mock catalogues are prepared for the $\Lambda$ CDM cosmological model with $\Omega_{m}=0.3, \Omega_{\Lambda}=0.7$ and with the amplitude of perturbations $\sigma_{8}=1.05$. For these catalogues the expected amplitude is $\tau_{m} / \sqrt{\Gamma} \approx 0.56$ and differences between measured and expected amplitudes characterize the actual precision achieved with this approach.

The richness and geometry of the DURS and LCRS are very different. Thus, DURS is an actual 3D catalogue but it contains $\sim 2500$ galaxies at the distance $D \leq 250 \mathrm{~h}^{-1} \mathrm{Mpc}$ and its representativeness is strongly limited. The LCRS includes $\sim 21000$ galaxies at the distance $D \leq 450 \mathrm{~h}^{-1} \mathrm{Mpc}$ but they are distributed within six thin slices that again distort the measured wall characteristics. Moreover, both catalogues include a small number of walls with large scatter in richness. This leads to a significant scatter of measured characteristics of walls for these catalogues which is a manifestation of well known cosmic variance. Only with an actually representative catalogue such as the the SDSS can this effect can be suppressed.

The scatter of the mean wall characteristics measured for the four samples of the DR1 reflects real variations of wall properties in these samples. It partially includes the dispersions depending on the shape of their PDFs. The actual scatter of the mean characteristics of walls averaged over all four samples of the DR1 is $\sim 10-12 \%$.

For the DR1 sample, the amplitude, $\tau_{\mathrm{m}}$, measured in the radial direction is

$$
\begin{aligned}
\tau_{\mathrm{m}} & \approx(0.53 \pm 0.05) \sqrt{\Gamma}=(0.24 \pm 0.02) \sqrt{\frac{\Gamma}{0.2}} \\
\sigma_{8} & \approx(1 \pm 0.1) \sqrt{\frac{\Gamma}{0.2}}
\end{aligned}
$$

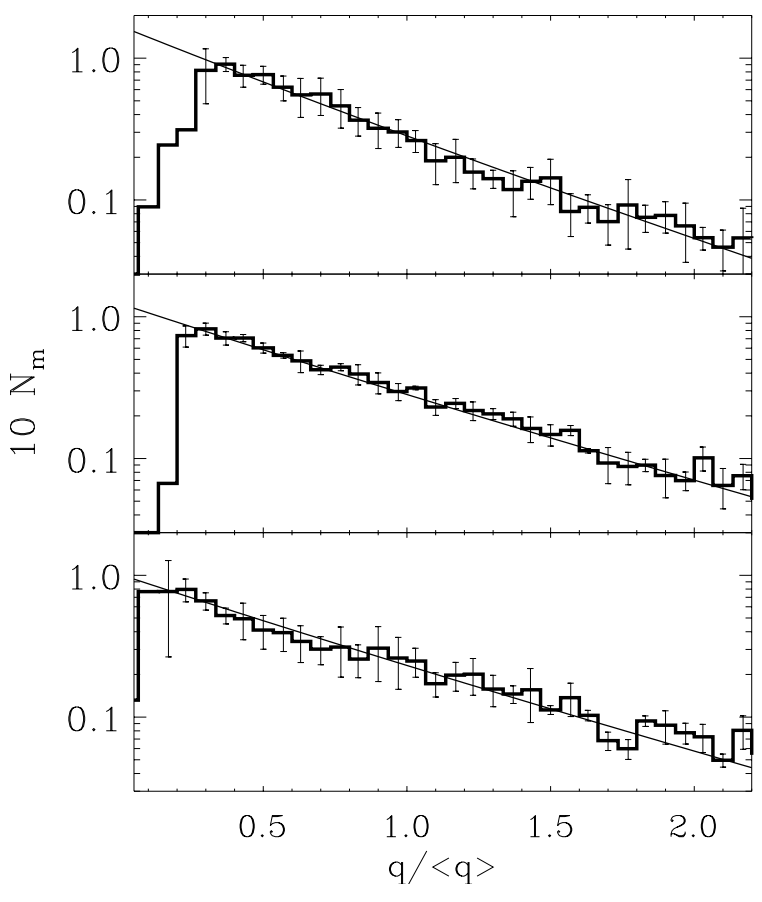

Fig. 10. The PDFs of dimensionless surface density of walls, $N_{\mathrm{m}}(q /\langle q\rangle)$, for walls selected in the DM simulation (top panel) and the mock catalogue in real (middle panel) and redshift (bottom panel) spaces. Fits (15) are plotted by solid lines.

The precision of this estimate is quite consistent with that found for the Cole et al. (1998) mock catalogue. Differences between this value and $\tau_{\mathrm{m}}$ measured in the transverse direction for the DR1, the LCRS and the DURS demonstrate the impact of the representativity of the catalogue used.

The measured PDFs of the surface density of walls are plotted in Figs. 10 and 11 for the simulated DM distribution, 


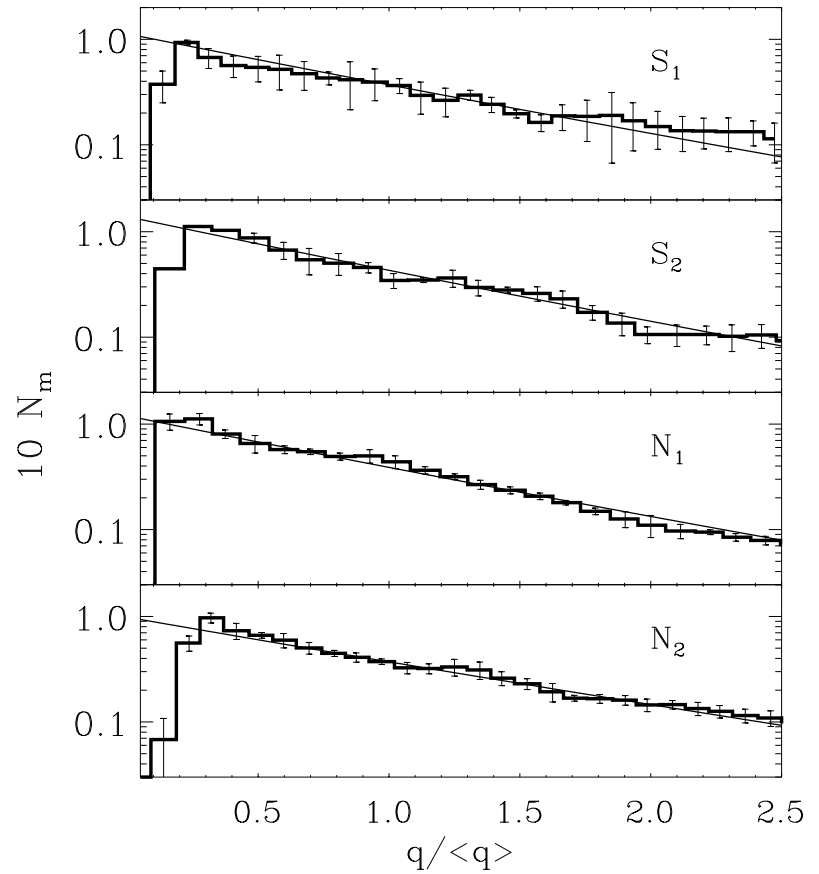

Fig. 11. The PDFs of observed dimensionless surface density of walls, $N_{m}(q /\langle q\rangle)$, for walls selected in four regions of the SDSS galaxy catalogues. Theoretically expected fits are plotted by solid lines.

mock catalogues in real and redshift spaces, and four observed samples of the SDSS DR1. These are nicely fit by the expected expression (15). Thus, for simulated samples we have, respectively,

$N_{\mathrm{m}}=(1 \pm 0.1) N_{\mathrm{th}}, \quad\left\langle q_{\mathrm{w}}\right\rangle /\left\langle q_{\mathrm{th}}\right\rangle=0.78,0.87,0.87$,

where $N_{\text {th }}$ and $\left\langle q_{\text {th }}\right\rangle$ are given by (15). For the two southern samples of the SDSS DR1 we get:

$N_{\mathrm{m}}=(1.1 \pm 0.2) N_{\mathrm{th}}, \quad\left\langle q_{\mathrm{w}}\right\rangle /\left\langle q_{\mathrm{th}}\right\rangle=1.07$,

and for the two northern samples of the SDSS DR1 we get:

$N_{\mathrm{m}}=(1 . \pm 0.1) N_{\mathrm{th}}, \quad\left\langle q_{\mathrm{w}}\right\rangle /\left\langle q_{\mathrm{th}}\right\rangle=0.97$.

These results verify that, the observed walls represent recently formed Zel'dovich pancakes.

The difference between the wall thickness measured in the radial and transverse directions, $h_{\mathrm{r}}$ and $h_{\mathrm{t}}$, indicates that along a short axis the walls are gravitationally confined stationary objects. Just as with the "Finger of God" effect for clusters of galaxies, this difference characterizes the gravitational potential of compressed DM rather than the actual wall thickness. The same effect is seen as a difference between the wall overdensities measured in radial and transverse directions.

The difference between the wall thicknesses is compared with the velocity dispersions of galaxies within the walls, $\left\langle w_{\mathrm{w}}\right\rangle$. Clusters of galaxies with large velocity dispersions incorporated in walls also increase the measured velocity dispersion. The correlation between the wall surface density and the velocity dispersion confirms the relaxation of matter within walls. This relaxation is probably accelerated due to strong small scale clustering of matter within walls.

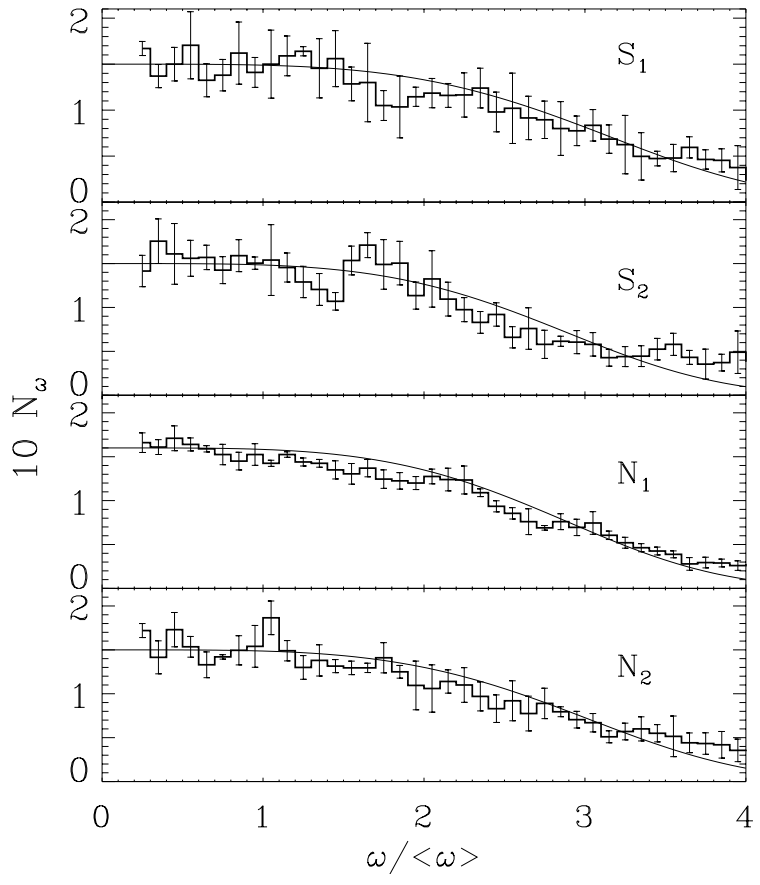

Fig. 12. The PDFs, $N_{\mathrm{w}}(w /\langle w\rangle)$, of reduced velocity dispersion within wall (25) for walls selected in four regions of the SDSS galaxy catalogues. Gaussian fits are plotted by solid lines.

Using the measured mean wall overdensity in the transverse direction listed in Table 1 we have for the parameter $\Theta_{\Phi}$ introduced in Eq. (19)

$\Theta_{\Phi} \approx \frac{\langle\delta\rangle}{3} \frac{0.3}{\Omega_{\mathrm{m}}} \approx 1.1$

which is also consistent with the expected values for relaxed and stationary walls.

As was proposed in Demiański et al. (2000) we can discriminate between systematic variations in the measured velocity dispersion due to regular variations in the surface density along the walls (Fig. 11) and the random variations in the velocity dispersion which integrates the evolutionary history of each wall. Indeed, along a shorter axis, for gravitationally bound and relaxed walls we can expect from Eq. (19) that

$w_{\mathrm{w}}^{2} \propto q_{\mathrm{w}}^{2} / \delta \propto \delta^{\gamma-1}, \quad \delta \propto q_{\mathrm{w}}^{2 / \gamma}, \quad w_{\mathrm{w}} \propto q_{\mathrm{w}}^{1-1 / \gamma}$.

Here we assume that the distribution of the DM component and galaxies can be approximately described by the polytropic equation of state with the power index $\gamma \approx 5 / 3-2$. Demiański et al. (2003) suggest for consideration a reduced velocity dispersion, $\omega_{\mathrm{w}}$,

$\omega_{\mathrm{w}}=\left|\ln \left(w_{\mathrm{w}} q_{\mathrm{w}}^{-p_{\mathrm{w}}}\right)\right|, \quad p_{\mathrm{w}} \approx 1-1 / \gamma \approx 0.5$,

corrected for variations of the wall thickness. For this function the systematic variations of $w_{\mathrm{w}}$ are essentially suppressed and, in most respects, it is similar to the entropy of compressed matter. It integrates the action of random factors in the course of wall formation. Hence, for this function the Gaussian PDF, $N_{\omega}$, can be expected. Indeed, this PDF plotted in Fig. 12 for four samples of the SDSS DR1 is quite similar to a Gaussian 


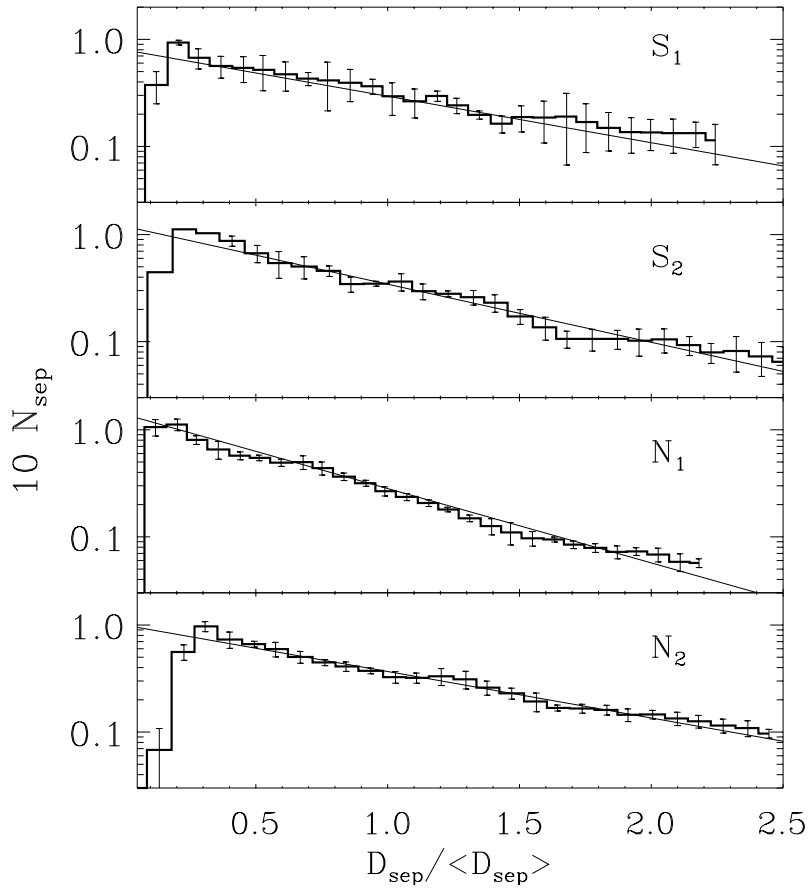

Fig. 13. The PDFs, $N_{\text {sep }}\left(D_{\text {sep }} /\left\langle D_{\text {sep }}\right\rangle\right)$, of observed wall separations for walls selected in four regions of the SDSS galaxy catalogues. Theoretically expected for the Poissonian distribution exponential fits are plotted by solid lines.

function with a standard deviation $\sigma_{\omega} \approx 2.25\langle\omega\rangle$. These results show a large scatter of evolutionary histories of observed walls.

Note that, for all the samples listed in Table 1, the mean wall separation, $\left\langle D_{\text {sep }}\right\rangle$, is close to twice that of the coherent length of the initial velocity field,

$\left\langle D_{\text {sep }}\right\rangle \approx 2 l_{v}$,

for the low density cosmological models with $\Gamma \approx 0.2$ (14). These results coincide with the estimates of the matter fraction, $\sim 50 \%$, accumulated within walls. Due to the large separation of walls, the correlations of their positions is small and a random 1D Poissonian PDF of the separation can be expected. These PDFs are plotted in Fig. 13 together with the exponential fits.

Finally, we would like to draw attention to the fact that all measured properties of these walls are quite consistent with a CDM-like initial power spectrum and Gaussian distribution of perturbations.

\section{Possible rich clusters of galaxies}

The SDSS DR1 also contains a number of galaxy complexes of various richnesses which can be extracted by means of the MST technique. Due to the large velocity dispersion of galaxies within clusters and the strong "Finger of God" effect, this extraction must be performed using different threshold linking lengths in the radial $\left(l_{\mathrm{r}}\right)$ and in the transverse $\left(l_{\mathrm{t}}\right)$ directions. This is not unlike how group catalogs are extracted from redshift surveys using conventional "friends-of-friends" algorithms (Huchra \& Geller 1982; Tucker et al. 2000).

We performed this cluster-finding in two major steps. First, we projected the observed samples onto a sphere of radius
$R=100 h^{-1} \mathrm{Mpc}$ with a random scatter $\pm 0.5 h^{-1} \mathrm{Mpc}$ and extracted a set of candidate clusters from this catalog using a linking length of $r_{\mathrm{t}}=0.3 \mathrm{~h}^{-1} \mathrm{Mpc}$. Second, we applied a radial linking length of $r_{\mathrm{r}}=3 \mathrm{~h}^{-1} \mathrm{Mpc}$ to these candidate clusters using their real $3 \mathrm{D}$ coordinates. In this second step, we also employed the threshold richness, $N_{\text {mem }}=10$, for our final samples of possible rich complexes. Having extracted these probable rich "clusters", we calculated a distance-independent measure of their richnesses by correcting their observed richnesses $N_{\text {mem }}$ for radial selection effects using Eq. (1); we call this corrected richness $N_{\text {sel }}$. Further discrimination of the "clusters" can be performed using their size in transverse and radial directions as well as other characteristics of selected galaxies.

For the threshold parameters used 20, 12, 43 and 10 possible rich clusters were selected from the $S_{1}, S_{2}, N_{1}$ and $N_{2}$ samples, respectively. The majority of these "clusters" are embedded within richer walls. Let us remember that these are possible rich clusters of galaxies and to confirm that they are physical potential wells, it is necessary to check for diffuse X-ray emission. However, comparison with the list from the NORAS survey (Böhringer et al. 2000) shows that only $\sim 32$ of them can be roughly related to the X-ray sources. This means that the method of cluster identification must be essentially improved. It can be expected that the possible incompleteness of the sample and especially rejection of brighter galaxies hamper the cluster identification. Perhaps, better results can be achieved with the richer photometric surveys of the SDSS.

\section{Mass function of the structure elements}

The richness of the SDSS DR1 allows one to extract several different sets of high density clouds and structure elements with various overdensities within the HDRs and LDRs and to find their mass function. These results can be directly compared with the theoretical expectations of DD02. However, the richness of the SDSS DR1 does not yet allow one to estimate quantitatively the divergence between the expected and observed mass functions plotted in Fig. 14.

Two samples of high density galaxy groups and two samples of unrelaxed structure elements - walls and filaments were selected separately within the HDRs and LDRs introduced in Sect. 3.3 for a threshold richness $N_{\text {mem }} \geq 5$. Since the velocity dispersions in groups are expected to be much smaller than those in rich clusters, we select these samples of structure elements using the simpler method described in Sect. 4 rather than the two-step approach described in Sect. 6. The richness of each group was corrected for radial selection effects using the selection function introduced in Sect. 2.1.

The main properties of the selected clouds are listed in Table 2, where $r_{\text {lnk }}$ and $\langle\delta\rangle$ are, respectively, the threshold linking length and the mean overdensity of clouds, $f_{\text {gal }}$ is the fraction of galaxies from the total (combined HDR+LDR) sample of galaxies within the selected clouds, $N_{\mathrm{cl}}$ is the number of clouds while $\langle M\rangle$ and $\left\langle M_{\text {sel }}\right\rangle$ are the observed and corrected for selection effect mean richness of individual clouds.

Results listed in Table 2 illustrate the influence of environment on the properties of high density clouds. In particular, in spite of the approximately equal number of galaxies in 


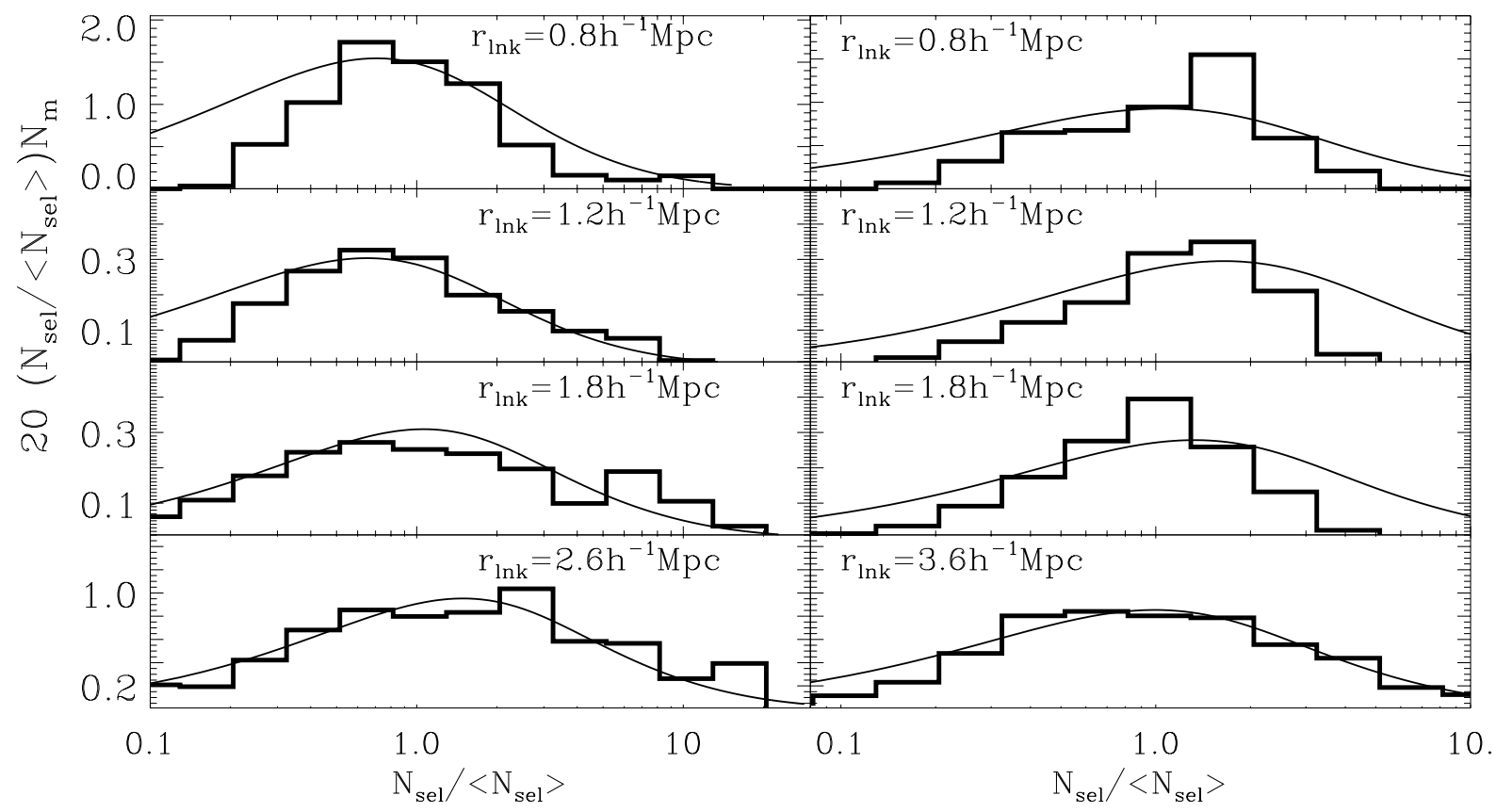

Fig. 14. Mass functions of galaxy clouds, $N_{\mathrm{m}} \cdot N_{\mathrm{mem}} /\left\langle N_{\mathrm{mem}}\right\rangle$ ), selected in HDRs (left panels) and LDRs (right panels) for four threshold linking lengths. Theoretical fit (27) for relaxed clouds $\left(r_{\mathrm{Ink}}=0.8\right.$ and $\left.1.2 h^{-1} \mathrm{Mpc}\right)$ and fit (28) for unrelaxed clouds $\left(r_{\mathrm{lnk}} \geq 1.8 h^{-1} \mathrm{Mpc}\right)$ are plotted by solid lines.

HDRs and LDRs, $\sim 85 \%$ and $\sim 70 \%$ of the high density clouds selected with linking lengths $r_{\text {lnk }}=0.8$ and $1.2 h^{-1} \mathrm{Mpc}$ are situated within the HDRs and accumulate $\sim 90 \%$ and $\sim 80 \%$ of galaxies. At linking length $r_{\text {lnk }}=1.8 h^{-1} \mathrm{Mpc}$ the numbers of clouds selected within HDRs and LDRs are comparable but again $\sim 80 \%$ of galaxies related to these clouds are concentrated within HDRs. At the largest linking lengths listed in Table 2 essential differences are seen only for the mean masses and number of structure elements selected within HDRs and LDRs. These differences are enhanced by the influence of the selection effect which is stronger for the LDRs.

The mass functions for these samples are plotted in Fig. 14. As was shown in DD02, in Zel'dovich theory and for the WDM initial power spectrum the dark matter mass function of structure elements is independent of their shapes and, at small redshifts, it can be approximated by the functions

$$
\begin{aligned}
& x N(x) \mathrm{d} x=12.5 \kappa_{\mathrm{ZA}} x^{2 / 3} \exp \left(-3.7 x^{1 / 3}\right) \operatorname{erf}\left(x^{2 / 3}\right) \mathrm{d} x, \\
& x N(x) \mathrm{d} x=8 . \kappa_{\mathrm{ZA}} x^{1 / 2} \exp \left(-3.1 x^{1 / 3}\right) \operatorname{erf}\left(x^{3 / 4}\right) \mathrm{d} x . \\
& x=\mu_{\mathrm{ZA}} \frac{M}{\langle M\rangle} .
\end{aligned}
$$

The expression (27) relates to clouds which have become essentially relaxed and static by $z=0$, and the expression (28) relates to richer, unrelaxed filaments and walls which are still in the process of collapse. Here, $\kappa_{\mathrm{ZA}} \sim 1.5-4$ and $\mu_{\mathrm{ZA}} \sim 0.8-1.3$ are fit parameters which take into account the incompleteness of selected samples of clouds for small and large richnesses; this incompleteness changes both the amplitude and mean mass of the measured clouds. Comparison with simulations (DD02) has shown that these relations fit reasonably well to the mass distribution of DM structure elements.
Table 2. Parameters of groups of galaxies selected in HDRs and LDRs after correction for the selection effect.

\begin{tabular}{crlrrr}
\hline \hline$r_{\text {Ink }} h^{-1} \mathrm{Mpc}$ & $\langle\delta\rangle$ & $f_{\text {gal }}$ & $N_{\mathrm{cl}}$ & $\langle M\rangle$ & $\left\langle M_{\text {sel }}\right\rangle$ \\
\hline & & HDR & & & \\
0.8 & 140 & 0.1 & 1193 & 6.8 & 12 \\
1.2 & 40 & 0.24 & 1961 & 9.5 & 25 \\
1.8 & 10 & 0.39 & 1731 & 17.7 & 61 \\
2.6 & 3 & 0.47 & 614 & 61.5 & 228 \\
\hline & & LDR & & & \\
0.8 & 220 & 0.014 & 220 & 4.8 & 14 \\
1.2 & 61 & 0.05 & 817 & 5.3 & 18 \\
1.8 & 16 & 0.15 & 1805 & 6.4 & 36 \\
3.6 & 3 & 0.36 & 2295 & 12.5 & 106 \\
\hline
\end{tabular}

These relations are similar to the mass function from the Press-Schechter formalism,

$x N_{\mathrm{PS}}(x) \mathrm{d} x=\frac{8 \kappa_{\mathrm{PS}}}{45 \sqrt{\pi}} \xi^{1 / 6} \exp \left(-\xi^{1 / 3}\right) \mathrm{d} x$,

$\xi=1.785 \mu_{\mathrm{ps}} x=1.785 \mu_{\mathrm{ps}} M /\langle M\rangle$,

despite the fact that they use different assumptions about the process of cloud formation and the shape of the formed clouds. Here again the fitting parameters $\kappa_{\mathrm{PS}}$ and $\mu_{\mathrm{PS}}$ take into account the incompleteness of the measured sample. However, this expression relates to the CDM-like power spectrum without small scale cutoff which is linked, for example, with the finite mass of DM particles, and without correction for the survival probability. So, it describes only the massive part of the mass function.

Relations (27) and (28) characterize the mass distribution of dark matter clouds associated with the observed galaxy 
groups and massive structure elements. They are closely linked with the initial power spectrum and fit reasonably well the observed mass distribution. For $N_{\text {sel }} \leq\left\langle N_{\text {sel }}\right\rangle$ the incompleteness of the sample of selected clouds leads to rapid drops in the observed mass functions as compared with theoretical expectations. For filaments selected within LDRs at $r_{\text {lnk }} \leq 2$ the deficit of richer clouds is enhanced by the method of filament separation. However, for the largest linking lengths, $r_{\mathrm{lnk}}=2.6$ and 3.6 $h^{-1} \mathrm{Mpc}$, where the incompleteness and other distortions are minimal, the observed mass distribution is quite consistent with theoretical expectations.

\section{Summary and discussion}

Statistical analysis of large galaxy redshift surveys allows one to obtain the quantitative characteristics of the large scale galaxy distribution, which in turn can be related to the fundamental characteristics of the Universe and the processes of structure formation. The large homogeneous data set compiled in the SDSS DR1 also permits one to check the results from analysis of the LCRS and the DURS and to obtain more accurate and more representative estimates of the main basic characteristics of the Universe.

As was discussed in Sect. 2, we use 79183 galaxies in four fields at the distance $D \leq 420 h^{-1} \mathrm{Mpc}$ where the influence of radial selection is moderate. Richness and geometry of the sample allow us to obtain representative characteristics of the observed LSS. Its possible incompleteness and, in particular, the deficit of brighter nearby galaxies does not strongly influence the properties of richer LSS elements but, probably distorts more strongly the properties of possible rich clusters of galaxies as discussed in Sect. 6. The radial selection effect usual for magnitude limited catalogues restricts the actual depth of the survey but its influence can be successfully corrected with methods described in Sect. 2.2.

The spatial galaxy distribution for the $S_{1}$ samples is plotted in Fig. 15; galaxies in HDRs are highlighted.

\subsection{Main results}

The main results of our investigation can be summarized as follows:

1. The analysis performed in Sect. 3 with the MST technique confirms that about half of galaxies are situated within rich wall-like structures and the majority of the remaining galaxies are concentrated within filaments. This result confirms that the filaments and walls are the main structure elements in the observed galaxy distribution. Quantitative characteristics of walls and filaments presented in Sects. 35 validate this division of the LSS into these two subpopulations.

2. The main characteristics of wall-like structure elements, such as the overdensity, separation distance between walls, wall thickness, and the velocity dispersion within walls, were measured separately for radial and, for the SDSS DR1 equatorial stripes, in transverse directions. Comparison of

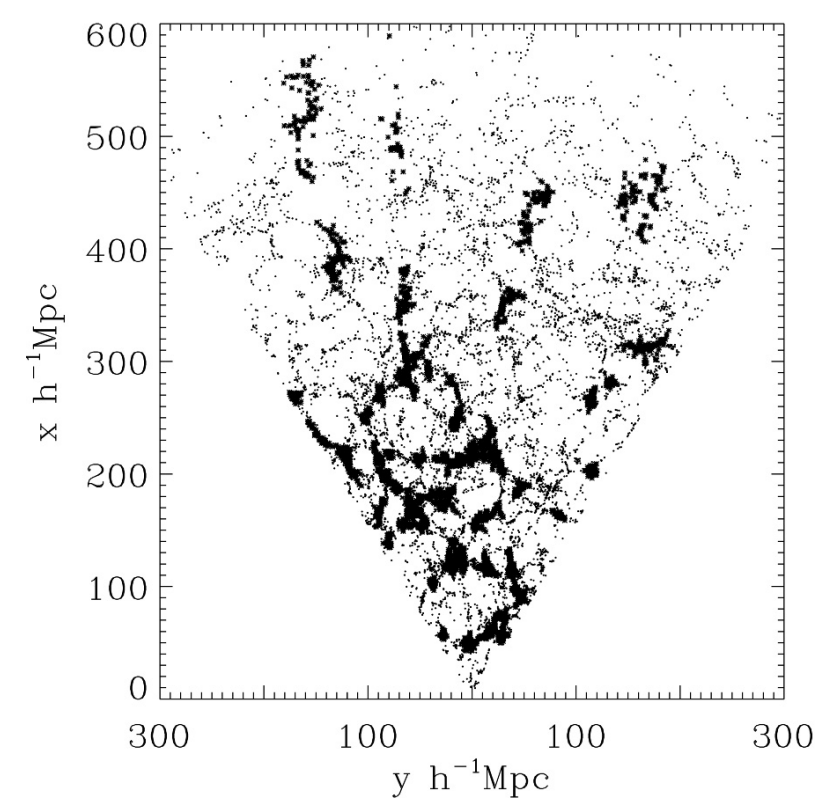

Fig. 15. Spatial distribution of all galaxies in the $S_{1}$ sample (points) and within the HDRs (thick points).

these characteristics demonstrates that the walls are approximately in static equilibrium, that they are relaxed along their shorter axis, and that their observed thickness in the radial direction is defined by the velocity dispersion of galaxies.

3. The PDF of the wall surface density is consistent with the simulated one and with that predicted by the Zel'dovich theory for Gaussian initial perturbations and the CDM-like initial power spectrum. The measured amplitude of perturbations coincides with that expected for a spatially flat $\Lambda \mathrm{CDM}$ cosmological model with $\Omega_{\Lambda} \approx 0.7$ and $\Omega_{\mathrm{m}} \approx 0.3$. These results demonstrate that the spatial galaxy distribution traces the dark matter distribution nicely.

4. The mass distributions of groups of galaxies, filaments and walls selected with various threshold overdensities nicely fit the joint mass function consistent with the expectations of Zel'dovich theory. Characteristics of these groups of galaxies listed in Table 2 clearly demonstrate the impact of environment and interaction of small and large scale perturbations.

5. We found the typical cell size of the filamentary network to be $\sim 10 h^{-1} \mathrm{Mpc}$ (Sect. 4). This estimate is consistent with the one obtained previously for the LCRS (Doroshkevich et al. 1996, 2001).

\subsection{Characteristics of the LSS}

Results obtained in Sect. 3 with the MST technique demonstrate that at least $80-90 \%$ of galaxies in the SDSS DR1 are concentrated within the filaments and walls with a variety of richness and overdensity forming the LSS. Comparison of the mean edge lengths $(6,8$ and 9) for the full sample, HDRs and LDRs with the mean density of the sample (3), shows that the LSS occupies only roughly half of the volume. It also shows that the mean overdensity of the walls is larger than 10 . 
The same results show that the filaments are formed by a system of high density groups of galaxies which contain $\approx 30 \%$ of all galaxies. For $\approx 20 \%$ of galaxies do we see the actual one dimensional PDF of the MST edge lengths (Fig. 5).

There is a continuous distribution in morphology and richness of the LSS elements. It can be roughly described as a system of walls randomly distributed in space with mean separations of $\approx(50 \pm 10) h^{-1} \mathrm{Mpc}$ and a filamentary network connected to the walls. The typical size of lower density regions, or voids, situated between filaments and walls is estimated in Sect. 4 as approximately $10-12 h^{-1} \mathrm{Mpc}$.

These results agree with those obtained for the mock catalogues simulating the galaxy distribution and indicate that galaxies nicely trace the spatial distribution of the dominant dark matter.

\subsection{Properties of walls and parameters of the initial power spectrum}

Walls and filaments are the largest structure elements observed in the Universe. In contrast to galaxies, their formation occurs at relatively small redshifts in course of the last stage of nonlinear matter clustering and is driven by the initial power spectrum of perturbations. Therefore, their properties can be successfully described by the nonlinear theory of gravitational instability (Zel'dovich 1970) that allows us to link them with the characteristics of the initial power spectrum.

The interpretation of walls as Zel'dovich pancakes has been discussed already in Thompson \& Gregory (1978) and in Oort (1983). The comparison of the statistical characteristics of the Zel'dovich pancakes for a CDM-like initial power spectrum (DD99, DD02) with those for observed walls demonstrates that, indeed, this interpretation is correct and, for a given cosmological model, it allows us to obtain independent estimates of the fundamental characteristics of the initial power spectrum.

The estimates of the mean wall surface density, $\left\langle q_{\mathrm{w}}\right\rangle$, and the amplitude of initial perturbations, $\left\langle\tau_{\mathrm{m}}\right\rangle$, listed in Table 1 are consistent with each other and with those found for the LCRS and DURS. They are also close to those found for the simulated DM distribution and for the mock galaxy catalogs (Cole et al. 1998) prepared for a spatially flat $\Lambda \mathrm{CDM}$ cosmological model with $\Omega_{\Lambda}=0.7, \Omega_{\mathrm{m}}=0.3$ and $\sigma_{8}=1.05$. As was shown in Sect. 5.3 (21), (22) and (23), the PDFs of both observed and simulated wall surface density plotted in Figs. 10 and 11 coincide with those theoretically expected (15) for Gaussian initial perturbations with a CDM-like power spectrum (DD99; DD02).

Averaging of both $\left\langle q_{\mathrm{w}}\right\rangle$ and $\left\langle\tau_{\mathrm{m}}\right\rangle$ listed in Table 1 allows us to estimate the mean values as follows:

$\left\langle q_{\mathrm{w}}\right\rangle=(0.38 \pm 0.06)(\Gamma / 0.2)$,

$\tau_{\mathrm{m}}=(0.24 \pm 0.02) \sqrt{\Gamma / 0.2} \quad \sigma_{8}=(1 . \pm 0.1) \sqrt{\Gamma / 0.2}$.

These values are consistent with the best estimates of the same amplitude summarized in Spergel et al. (2003) for the $\Lambda$ CDM cosmological model with $\Gamma=0.2$

$\sigma_{8} \approx 0.9 \pm 0.1, \quad \tau \approx 0.22 \pm 0.03$,
These results verify that galaxies nicely trace the LSS formed mainly by the dark matter distribution and the observed walls are recently formed Zel'dovich pancakes. They also verify the Gaussian distribution of initial perturbations and coincide with the Harrison-Zel'dovich primordial power spectrum.

Comparison of other wall characteristics measured in radial and transverse directions indicate that the walls are gravitationally confined and relaxed along the shorter axis. The same comparison allows us to find the true wall overdensity, wall thickness, and the radial velocity dispersion of galaxies within walls. As is seen from relation (19), these values are quite self-consistent.

\subsection{Mass function of structure elements}

The samples of walls, filaments, and groups of galaxies in the SDSS DR1 selected using different threshold overdensities allow us to measure their mass functions, to trace their dependence on the threshold overdensity and environment, and to compare them with the expectations of Zel'dovich theory.

This comparison verifies that for lower threshold overdensities for both filaments and wall-like structure elements, the shape of the observed mass functions is consistent with the expectations of Zel'dovich theory. However, for high density groups of galaxies some deficit of low mass groups caused, in particular, by selection effects and enhanced by the restrictions inherent in our procedure for group-finding leads to a stronger difference between the observed and expected mass functions for $M_{\text {sel }} \leq\left\langle M_{\text {sel }}\right\rangle$. The same factors distort the observed mass functions for groups selected within LDRs.

Let us note that mass functions (27, 28, and 29) are closely linked with the initial power spectrum. This is manifested as a suppression of the PDFs at $M_{\text {sel }} \leq\left\langle M_{\text {sel }}\right\rangle$ and is proportional to $\exp \left[-\left(M /\left\langle M_{\text {sel }}\right\rangle\right)^{1 / 3}\right]$ at $M_{\text {sel }} \geq\left\langle M_{\text {sel }}\right\rangle$. They differ from the mass function of clusters of galaxies and the probable mass function of observed galaxies which are formed on account of multi-step merging of less massive clouds and are described by a power law with a negative power index at $M_{\text {sel }} \leq\left\langle M_{\text {sel }}\right\rangle$ and an exponential cutoff $\propto \exp \left[-\left(M /\left\langle M_{\text {sel }}\right\rangle\right)\right.$ at $M_{\text {sel }} \geq\left\langle M_{\text {sel }}\right\rangle$ (see e.g. Silk \& White 1978).

\subsection{Interaction of large and small scale perturbations}

The data listed in Table 2 shows that the majority of high density groups of galaxies and the main fraction of galaxies related to these groups (up to 80-90\%) are situated within HDRs. These results illustrate the influence of environment on galaxy formation and the clustering of luminous matter. It also indicates the importance of interactions of small and large scale perturbations for the formation of the observed LSS. This problem was also discussed in Einasto et al. (2003a,b).

These differences are partly enhanced by the influence of selection effects. Indeed, the majority of HDRs are situated at moderate distances $D \sim 150-300 h^{-1} \mathrm{Mpc}$ where this influence is not so strong. On the other hand the LDRs also include all galaxies situated in the farthest low density regions. However, as was shown in DD02 significant differences between the 
characteristics of clouds separated within HDRs and LDRs is seen even for simulated clustering of dark matter.

These results are consistent with the high concentration of observed galaxies within the filaments and walls forming the LSS noted in Sect. 8.2. Bearing in mind that the mean density of luminous matter does not exceed $10 \%$ of the full matter density of the Universe we can conclude that galaxies are formed at high redshifts presumably within compressed regions which are now seen as elements of LSS.

A natural explanation for both of these differences and the high concentration of galaxies within the LSS elements is the interaction of small and large scale perturbations when large scale compression of matter accelerates the formation of small scale high density clouds. Such interactions may also explain the existence of large voids similar to the Böotes void where formation of galaxies has been strongly suppressed.

\subsection{Possible rich clusters of galaxies}

The MST technique generalizes the standard "friends-offriends" method of the selection of denser clouds and of probable clusters of galaxies. However, first attempts of such selection presented in Sect. 6 show that there is an essential difference between the selected high density clouds and X-ray sources. The nature of this difference is not yet clear and perhaps it will be eliminated after the introduction of stronger criteria for the selection of probable clusters of galaxies from the survey.

\subsection{Final comments}

The SDSS (York et al. 2000; Stoughton et al. 2002; Abazajian et al. 2003) and $2 \mathrm{dF}$ (Colless et al. 2001) galaxy redshift surveys provide deep and broad vistas with which cosmologists may study the galaxy distribution on extremely large scalesscales on which the imprint from the primordial fluctuation spectrum has not been erased.

In this paper, we have used the SDSS DR1 to investigate the galaxy distribution at such large scales. We have confirmed our earlier results, based on the LCRS and DURS samples, that galaxies are distributed in roughly equal numbers between two different environments: filaments, which dominate low density regions, and walls, which dominate high density regions. Although different in character, these two environments together form a fragmented joint random network of galaxies the cosmic web.

Comparison with theory strongly supports the idea that the properties of the observed walls are consistent with those for Zel'dovich pancakes formed from a Gaussian spectrum of initial perturbations for a flat $\Lambda$ CDM Universe $\left(\Omega_{\Lambda} \approx 0.7\right.$, $\Omega_{\mathrm{m}} \approx 0.3$ ). These results are consistent with the estimate of $\Gamma=0.20 \pm 0.03$ obtained in Percival et al. (2001) for the $2 \mathrm{dF}$ Galaxy Redshift Survey (see also Spergel et al. 2003).

Such analysis allows one to obtain some important basic conclusions regarding the properties and the process of the formation and evolution of the large scale structure of the
Universe. With future public releases of the SDSS data set, we hope to refine these conclusions.

Acknowledgements. We thank Shiyin Shen of the Max-PlanckInstitut für Astrophysik and Jörg Retzlaff of the Max-Planck-Institut für Extraterrestrial Physics for useful discussions regarding this work. Funding for the creation and distribution of the SDSS Archive has been provided by the Alfred P. Sloan Foundation, the Participating Institutions, the National Aeronautics and Space Administration, the National Science Foundation, the US Department of Energy, the Japanese Monbukagakusho, and the Max Planck Society. The SDSS Web site is http://www.sdss.org/

The Participating Institutions are the University of Chicago, Fermilab, the Institute for Advanced Study, the Japan Participation Group, the Johns Hopkins University, the Max Planck Institute for Astronomy (MPIA), the Max Planck Institute for Astrophysics (MPA), New Mexico State University, Princeton University, the United States Naval Observatory, and the University of Washington.

This paper was supported in part by Denmark's Grundforskningsfond through its support for an establishment of the Theoretical Astrophysics Center.

\section{References}

Abazajian, K., Adelman-McCarthy, J. K., Agüeros, M. A., Allam, S. S., \& the SDSS Collaboration 2003 [arXiv: astro-ph/0305492]

Böhringer, H., Voges, W., Huchra, J. P., et al. 2000, ApJS, 129, 435

Barrow, J. D., Bhavsar, S. P., \& Sonoda, D. H. 1985, MNRAS, 216, 17

Baugh, C. M., \& Efstathiou, G. 1993, MNRAS, 265, 145

Bhavsar, S. P., \& Ling, E. N. 1988, ApJ, 331, L63

Buryak, O., \& Doroshkevich, A. 1996, A\&A, 306, 1

Cole, S., Hatton, S., Weinberg, D. H., \& Frenk, C. S. 1998, MNRAS, 300, 945

Colless, M., Dalton, G., Maddox, et al. 2001, MNRAS, 328, 1039

Demiański, M., \& Doroshkevich, A. G. 1999, MNRAS, 306, 779

Demiański, M., \& Doroshkevich, A. G. 2002, [arXiv: astro-ph/0206282]

Demiański, M., Doroshkevich, A. G., \& Turchaninov, V. 2000, MNRAS, 318, 1189

Demiański, M., Doroshkevich, A. G., \& Turchaninov, V. 2003, MNRAS, 340, 525

Doroshkevich, A. G., Fong, R., McCracken, H. J., et al. 2000, MNRAS, 315, 767

Doroshkevich, A. G., Tucker, D. L., Fong, R., Turchaninov, V., \& Lin, H. 2001, MNRAS, 322, 369

Doroshkevich, A. G., Tucker, D. L., Oemler, A. J., et al. 1996, MNRAS, 283, 1281

Einasto, M., Einasto, J., Müller, V., Heinämäki, P., \& Tucker, D. L. 2003, A\&A, 401, 851

Einasto, J., Hútsi, G., Einasto, M., et al. 2003, A\&A, 405, 425

Eisenstein, D. J., Annis, J., Gunn, J. E., et al. 2001, AJ, 122, 2267

Fukugita, M., Ichikawa, T., Gunn, J. E., et al. 1996, AJ, 111, 1748

Gómez, P. L., Nichol, R. C., Miller, C. J., et al. 2003, ApJ, 584, 210

Goto, T., Okamura, S., Sekiguchi, M., et al. 2003, PASJ, 55, 757

Gunn, J. E., Carr, M., Rockosi, C., Sekiguchi, M., \& the SDSS Collaboration 1998, AJ, 116, 3040

Hoyle, F., Vogeley, M. S., Gott, J. R. III, et al. 2002, ApJ, 580, 663

Huchra, J. P., \& Geller, M. J. 1982, ApJ, 257, 423

Jenkins, A., Frenk, C. S., Pearce, F. R., et al. 1998, ApJ, 499, 20

Kruskal, J. B. 1956, Proc. Am. Math. Soc., 7, 48

Loeb, A., \& Barkana, R. 2001, ARA\&A, 39, 19 
Oort, J. H. 1983, ARA\&A, 21, 373

Percival, W. J., Baugh, C. M., Bland-Hawthorn, J., et al. 2001, MNRAS, 327, 1297

Prim, A. 1957, Bell System Tech. J., 36, 1389

Ratcliffe, A., Shanks, T., Broadbent, A., et al. 1996, MNRAS, 281, L47

Schmalzing, J., Gottlöber, S., Klypin, A. A., \& Kravtsov, A. V. 1999, MNRAS, 309, 1007

Shandarin, S. F., \& Zeldovich, Y. B. 1989, Rev. Mod. Phys., 61, 185

Shectman, S. A., Landy, S. D., Oemler, A., et al. 1996, ApJ, 470, 172

Sheth, J. V., Sahni, V., Shandarin, S. F., \& Sathyaprakash, B. S. 2002, [arXiv:astro-ph/0210136]

Silk, J., \& White, S. D. 1978, ApJ, 223, L59

Spergel, D. N., Verde, L., Peiris, H. V., et al. 2003, [arXiv:astro-ph/0302209]
Stoughton, C., Lupton, R. H., Bernardi, M., Blanton, M. R., \& the SDSS Collaboration. 2002, AJ, 123, 485

Strauss, M. A., Weinberg, D. H., Lupton, R. H., et al. 2002, AJ, 124, 1810

Tegmark, M., Blanton, M., Strauss, M., et al. 2003, [arXiv:astro-ph/10725]

Thompson, L. A., \& Gregory, S. A. 1978, ApJ, 220, 809

Tucker, D. L., Oemler, A. J., Hashimoto, Y., et al. 2000, ApJS, 130, 237

van de Weygaert, M. A. M. 1991, Ph.D. Thesis, Leiden: Rijksuniversiteit

York, D. G., Adelman, J., Anderson, J. E., Anderson, S. F., \& the SDSS Collaboration. 2000, AJ, 120, 1579

Zel'dovich, Y. B. 1970, A\&A, 5, 84 